\title{
Simulations of STEADY Flows THROUGH CYLINDRICAL GEOMETRIES WITH AND WITHOUT LOCAL CONSTRICTIONS BY MULTIPARTICLE COLLISION DYNAMICS
}

\author{
by \\ Salil Kishor Bedkihal \\ Masters of Science, University of Pune, India, 2003 \\ Bachelor of Science, University of Pune, India, 2001
}

\author{
A thesis \\ presented to Ryerson University \\ in partial fulfillment of the \\ requirements for the degree of \\ Master of Science \\ in the Program of \\ Biomedical Physics
}

Toronto, Ontario, Canada, 2010

(C)Salil Kishor Bedkihal 2010 
I hereby declare that I am the sole author of this thesis.

I authorize Ryerson University to lend this thesis to other institutions or individuals for the purpose of scholarly research.

I further authorize Ryerson University to reproduce this thesis by photocopying or by other means, in total or in part, at the request of other institutions or individuals for the purpose of scholarly research. 
Simulations of steady flows through cylindrical geometries with and without local constrictions by multiparticle collision dynamics

Master of Science 2010

Salil Kishor Bedkihal

Biomedical Physics

Ryerson University

In this thesis, a recently developed particle-based method called multiparticle collision dynamics $(M P C)$ is used to simulate steady flows through three-dimensional constricted axisymmetric cylinders. The work is motivated by complex particle interactions in blood flow such as aggregation and the need to be able to capture these effects in physiologically relevant complex flow geometries. This is the first time that $M P C$ dynamics has been applied to simulate flows through constrictions. The particle collisions in $M P C$ dynamics are numerically more efficient than other particle-based simulation methods. Particle interactions with the cylinder walls are modeled using bounce-back $(B B)$ and loss in tangential,reversal of normal (LIT) boundary conditions. $B B$ is an analog of the macroscopic no-slip boundary condition, and LIT gives slip. Finally, an averaging procedure is employed to make a connection with the solution to the Navier-Stokes equations. Interesting differences have been found in the velocity profiles obtained using $M P C$ with $B B$ and LIT, compared to Navier-Stokes. 


\section{Acknowledgements}

I would like to thank all those people who made this work possible. First of all I am thankful to my academic parents, my supervisors Dr. Rohlf and Dr. Kumaradas for their inexorable support. They gave me a lot of time and space to implement my own ideas. I am also thankful to the department of Mathematics and Physics for providing me the excellent computational resources. Further thanks to my family members Sharmila, Sudhir, my cousin Neeraja and my grandparents for their moral and emotional support during this work. I am very thankful to my friends especially Irina and Cristina for their support and long scientific debates. I have no words to describe what my father did for me. I am very thankful to him that he nurtured my scientific interests and helped to develop a faculty of critical thinking. I would like to extend my thanks to Dr. Raymond Kapral at University of Toronto, Department of Chemical Physics. I really enjoyed the scientific discussions with him. My supervisory committee members Dr. Nancy Ford and Dr. Catherine Beauchemin have played an important role during this work. I am always indebted to them for their critical comments. I am very thankful to my former supervisor and my hero, Dr. Pathak for his moral and emotional support. I am very greatful to Iosif Deac for giving me an opportunity to work as teaching assistant in senior undergraduate physics courses. The last but not the least are Leo Zambito and Khurrum who almost instantaneously responded and acted to resolve any computer issues. Finally I am thankful to NSERC for funding this research work and Ryerson University for giving me an opportunity to pursue a graduate degree in Biomedical physics program. 


\section{Dedication}

I would like to dedicate this thesis to my mother Savita who is not living now. She always motivated me to learn science, and especially Physics. 


\section{Contents}

1 Introduction $\quad 3$

1.1 Biological Motivations . . . . . . . . . . . . . . . . . . . . 3

1.2 Particle-Based Methods . . . . . . . . . . . . . . . . 5

1.3 Specific Aim And Methodology . . . . . . . . . . . . . . . 8

1.4 Thesis Organization . . . . . . . . . . . . . . . . . 9

2 Fluid Dynamics $\quad 10$

2.1 Chapter Overview . . . . . . . . . . . . . . . . . . . . 10

2.2 Continuum fluid dynamics . . . . . . . . . . . . . . . . . . 11

$2.3 M P C$ dynamics . . . . . . . . . . . . . . . . . . 13

2.3.1 Multiparticle collision . . . . . . . . . . . . . 13

2.3.2 Gravity-Driven Flow and Thermostatting . . . . . . . . . . . 16

2.3.3 Free-streaming, Liouville and $H$-Theorems . . . . . . . . . . . . . 17

2.3.4 Boundary conditions .................. 18

2.3.5 Grid-shifting. . . . . . . . . . . . . . . . . 19

2.4 Averaging Procedure . . . . . . . . . . . . . . . . . . . . . 19

2.5 Transport Coefficients . . . . . . . . . . . . . . . . . 20

2.6 Finite element simulation . . . . . . . . . . . . . . . . . . . 22

2.7 Geometry and MPC Parameters . . . . . . . . . . . . . . . 22

3 Results and Discussion $\quad 25$

3.1 Poiseuille flow . . . . . . . . . . . . . . . . . . . 25

$3.2 R e \approx 4, g=0.0025, s_{c}=0.2 \ldots \ldots \ldots . \ldots \ldots$

$3.3 R e \approx 7, g=0.005, s_{c}=0.2 \ldots \ldots \ldots \ldots$

$3.4 R e \approx 15, g=0.01, s_{c}=0.2 \ldots \ldots . \ldots . \ldots . \ldots 29$ 
3.5 Additional tests . . . . . . . . . . . . . . . . . . . . . . 32

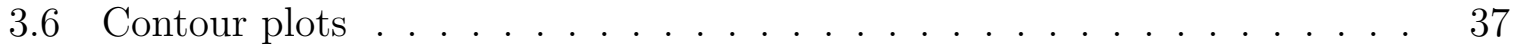

3.7 LIT Comparison . . . . . . . . . . . . . . . . . . . . . . . . . . . 37

3.8 Summary of Key Findings . . . . . . . . . . . . . . . . . . 43

3.9 Discussion . . . . . . . . . . . . . . . . . . . . . . . . 47

4 Conclusion and Future Work 49

4.1 Summary . . . . . . . . . . . . . . . . . . . . . . . . . . . . . . 49

4.2 Conclusion . . . . . . . . . . . . . . . . . 50

4.3 Future Work . . . . . . . . . . . . . . . . . . . . . . . 51

$\begin{array}{ll}\text { References } & 57\end{array}$ 


\section{List of Tables}

2.1 Simulation Parameters . . . . . . . . . . . . . . . . . . . . . 23

3.1 Local Mach numbers in the constriction . . . . . . . . . . . . . . 48 


\section{List of Figures}

2.1 Diagrammatic representation of the multiparticle collision rule. The upper pannel shows the center of mass velocity and the pre-collision velocities of two particles relative to the center of mass. The lower pannel shows the result of adding back the center of mass velocities to get post-collision velocities. The figure is adapted from Anatoly Malevanets and Raymond Kapral $[1]$. . . . . . . . . . . . . . . . . . . . . . . . . . . . . . . . . 15

2.2 Flow Geometry ...................... . . 23

3.1 Cross-section velocity profiles for Poiseuille flow with $\mathrm{Re}=7$. . . . . . . . 26

3.2 Contour velocity plots for Poiseuille flow using $M P C$ with $\operatorname{Re}=7$ using (A) LIT and (B)BB boundary conditions. . . . . . . . . . . . . . . 27

3.3 Centerline velocity profiles for 40 percent stenosis with $\mathrm{Re}=4$. . . . . . . 29

3.4 Cross-section velocity profiles upstream of the 40 percent stenosis at A with $\mathrm{Re}=4 \ldots \ldots . \ldots$. . . . . . . . . . . . . . . 30

3.5 Cross-section velocity profiles downstream of the 40 percent stenosis at B with $\mathrm{Re}=4$. . . . . . . . . . . . . . . . . . . . . . 31

3.6 Centerline velocity profiles for 40 percent stenosis with $\mathrm{Re}=7$. . . . . . . 32

3.7 Cross-section velocity profiles upstream of the 40 percent stenosis at A with $\mathrm{Re}=7 \ldots \ldots \ldots \ldots \ldots \ldots \ldots$

3.8 Cross-section velocity profiles downstream of the 40 percent stenosis at B $: \operatorname{Re}=7$.

3.9 Cross-section density profiles $(\mathrm{A})$ upstream of $y_{c}=226.5$ at point $\mathrm{A}$ and (B) downstream at point B for the 40 percent stenosis :Re $=7$. . . . . . 35

3.10 Radial velocity plot upstream of the 40 percent constriction at $\mathrm{A}: \mathrm{Re}=7 \quad 36$

3.11 Radial velocity plots downstream of the 40 percent constriction at $\mathrm{B}: \mathrm{Re}=736$ 
3.12 Centerline velocity profiles for 40 percent stenosis : Re=15 . . . . . . . . 37

3.13 Cross-section velocity profiles upstream of the 40 percent stenosis at $\mathrm{A}$ : $\mathrm{Re}=15 \ldots \ldots \ldots \ldots \ldots \ldots$

3.14 Cross-section velocity profiles downstream of the 40 percent stenosis at B : $\mathrm{Re}=15 \ldots \ldots \ldots \ldots$. . . . . . . . . . . . . . . . . . . . . . . . .

$3.15 x$-component of momentum as a function of time . . . . . . . . . . 40

$3.16 y$-component of momentum as a function of time . . . . . . . . . . . . 40

$3.17 z$-component of momentum as a function of time . . . . . . . . . . . 41

3.18 Temperature as a function of time . . . . . . . . . . . . . . . . . 41

3.19 Velocity contour plots, (A) using LIT and (B) using BB boundary conditions with a 40 percent constriction : $\mathrm{Re}=7$. . . . . . . . . . . 42

3.20 Centerline velocity profiles for LIT, 40 percent stenosis . . . . . . . . . . 43

3.21 Centerline velocity profiles in case of LIT : Re=7 . . . . . . . . . . . . 44

3.22 Scaled density along centerline as a function of scaled axial coordinate for

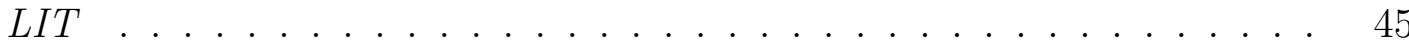

3.23 Scaled density along centerline as a function of scaled axial coordinate for the $B B$ rule . . . . . . . . . . . . . . . . . . . . . . . . . . . . . . . . . 46 


\section{Notations}

$\widehat{\omega} \quad$ Stochastic rotation operator

$\nabla \quad$ Gradient operator

$\xi \quad$ Collision cell number

$\mathbf{v}(r=0) \quad$ Centerline velocity in finite element simulations (comsol Multiphysics) notation

$U_{c} \quad$ Centerline velocity in unconstricted portion of the cylinder

$<\mathbf{u}_{\xi}>$ Average flow velocity in $M P C$ dynamics

$<\mathbf{u}_{\xi}(0)>_{y} \quad$ Centerline velocity in $M P C$ dynamics

$<\mathbf{u}_{\xi}>_{z} \quad z$-component of velocity

$L_{x} \quad$ Number of collision cells along $x$ direction

$L_{y} \quad$ Number of collision cells along $y$ direction

$L_{z} \quad$ Number of collision cells along $z$ direction

$D$ Diameter of the unconstricted cylinder

$s_{c} \quad$ Severity of constriction

$L \quad$ Length of the stenosis

$k_{B} \quad$ Boltzmann constant

$T$ System temperature

$y_{c} \quad$ Center of the stenosis

$m \quad$ Particle mass

$\mathbf{v}_{i}^{\prime \prime} \quad$ Post-collision velocity of particle $i$

$\mathbf{v}_{i}^{\prime} \quad$ Pre-collision velocity of particle $i$ 
$\mathbf{V}_{\xi}^{\prime} \quad$ Average cell velocity

p Momentum vector

$\mu$ Dynamic viscosity

$\nu \quad$ Kinematic viscosity

$\rho$ Density

$n \quad$ Average number of particles in a cell

$n^{*} \quad$ Number of particles along the centerline scaled by $n$

Re Reynolds number

Ma Mach number

$g$ Parameter in gravity driven flow

F Force 


\section{Chapter 1}

\section{Introduction}

\subsection{Biological Motivations}

The complex nature of blood flow can largely be attributed to the cellular components of blood and the complex physiological geometries. In a $1 \mathrm{~mm}^{3}$ volume of blood, there are millions of red blood cells (RBCs) that interact with each other and can stick together to form larger clusters in a process called aggregation. In flow, the shear-dependent aggregation and break-up of RBC clusters is believed to lead to non-Newtonian flow behaviour. The RBC aggregates can create a solid core, and in order to break up this core for blood to flow, a minimum amount of stress is required. This minimum stress is known as the yield stress [2] and is a non-Newtonian property. Whether or not blood has a yield stress is a controversial issue that needs to be resolved. The experimental determination of yield stress is based on extrapolation procedures, and hence their accuracy also depends on the particular extrapolation procedure used. Particle-based simulation methods can be used to shed light on the yield stress issue.

In the process of RBC aggregation and break-up, the total number of clusters before and after the break-up need not be conserved. The fluctuations in particle numbers can not be handled by the traditional kinetic theory descriptions in the form of the Boltzmann equation. In this case the reactive multiparticle collision dynamics is an appropriate formalism. The RBC aggregation and break-up can be treated like chemical reactions where reaction rates now depend on the shear rate. Rohlf has extended multiparticle collision dynamics to chemical reactions where the total number of particles can change 
in time [3]. The reaction rates used in this work are constant and the case of no flow is considered. It has been shown that with an appropriate averaging procedure, macroscopic rate laws for chemical reactions can be recovered.

Complex geometries, such as local constrictions in blood vessels, can also cause RBC aggregation. The ultrasonic measurements of RBC aggregation in constricted tubes have been reported [4] and it was found that RBCs form clusters in the post-constriction region. In vitro experiments have been performed to investigate haemodynamic and haemorheological behaviours of hameodiluted blood flow through microstenosis using a micro-particle image velocimetry (PIV) method [5]. In addition to reported RBC aggregation, it was found that the mean velocity of blood was symmetric with respect to the channel centerline and in the contraction region of the channel. Additionally, the maximum velocity in the constriction was found to be 5 times greater than that of the straight channel without stenosis [5]. Rolling, tumbling and twisting motions of red blood cells were also observed due to the flow choking characteristics in the stenotic region [5]. These aspects can only be captured by using discrete particle-based simulation methods.

Flows through constrictions are of primary importance in physiology. In general, flow speeds up through the constriction, and decreases once it has passed through. The sudden increase of flow speed gives rise to adverse pressure gradients, causing low shear regions near the vessel wall. Platelets trapped in low shear zones form aggregates and adhere to the vessel wall leading to thrombus (or clot) formation. The dynamics of blood flow through constricted channels has been studied numerically $[6,7,8]$ and experimentally [9] by several researchers. In most of the published numerical work, flow behaviour is investigated using no-slip boundary conditions where fluid velocity is zero at the solid boundaries.

Nubar [10] has suggested the possibility of slip for blood in viscometers. Hershey and Cho performed an experimental study of blood flow in rigid tubes [11]. They found that the thickness of the plasma film increased rapidly with increasing flow rate, and that the effective slip velocity of the plasma film also increased monotonically with the flow rate and was a linear function of shear stress at high flow rates. Velocity profiles of blood flow in rat mesentery arterioles with bifurcation and confluence have been measured by particle image velocimetry by Nakano et. al [12]. In this experiment, images of red blood cells in flow were recorded. The time averaged cross-section velocity profiles obtained in this experiment showed an appearance of slip near the vascular wall. Misra and Shit [13] 
have performed a numerical study of blood flow through stenosed arteries using a nonNewtonian model. Their results show an enhancement in volumetric flow rate with an increase in slip velocity. Picart et.al [14] have reported the slip at the walls in viscometric measurements of the yield stress of the blood. Based on these reports, the possibility of slip in case of blood flow studies can not be ruled out. In case of flows through stenosis with slip at the wall, the flow rate would be higher than that of the no-slip case. If a drug is injected in the blood stream so that it increases the slip at the wall [15], then even in the presence of stenosis, one could recover a proper flow rate of blood. The wall shear stress in case of no-slip would be higher than that of slip. So by injecting a drug as mentioned above, one could minimize the risk of plaque rupture as well.

The slip can also be incorporated in the Navier-Stokes equations. Since blood is a suspension of cells and complex geometries like stenosis affect the cell interactions that in turn affect the flow dynamics, discrete particle-based methods that are capable of incorporating slip boundary conditions are an appropriate choice to capture these mesoscale processes. This way the effect of slip on the particle interactions can also be studied.

\section{$1.2 \quad$ Particle-Based Methods}

Computer simulations play a very important role in our understanding of complex systems. They act as a virtual laboratory and also allow us to validate experimental data. There are many fields where computer simulations are essential. They include dynamics of biomolecules, equilibrium and non-equilibrium transport phenomena, virology, electronic structure calculations, astrophysical processes and many more. One of the most challenging applications of computer simulations are fluid systems, as the structure and dynamics of complex fluids comprises many phenomena that are not very well understood. The most challenging task in simulations of complex fluids is to capture the diverse spatial and temporal scales.

In colloidal suspensions a typical micron sized particle diffuses over the length of its diameter in one second but displaces the billions of solvent molecules in a few picoseconds. The multiscale hierarchy of the blood circulatory system is another example of a complex flow system. Depending on the diameter of the vessel, blood shows different behaviour. 
In larger arteries blood can be regarded as a continuum, while in capillaries, where the diameter is of the order of the red blood cell, particulate effects dominate and the continuum approximation is no longer appropriate.

Many phenomena of interest in complex fluids appear at time scales much larger than the motion of individual solvent molecules. In this case, irrelevent microscopic degrees of freedom need to be removed and only relevant variables, that evolve on a suitable time scale, are retained. This procedure is called coarse graining and is the basis of discrete particle-based simulation methods.

Today, a wide variety of discrete particle-based simulation methods exist for complex flow phenomena. These methods can be classified into two main categories, namely lattice and off-lattice methods. Lattice Boltzmann and lattice gas automata are two popular lattice-based simulation methods. In these methods, point-like particles reside on a regular lattice where they move from node to node and undergo collisions only when their trajectories meet. These collisions occur under simple collision rules. As long as these collision rules obey symmetry and invariance principles, correct hydrodynamic behaviour can be recovered at macroscopic scales. These methods are easy to implement and computationally inexpensive. A major drawback of lattice-based methods is that the dynamics are constrained by the configuration of the lattice [1].

The off-lattice particle-based methods include dissipative particle dynamics (DPD), direct simulation Monte-Carlo methods (DSMC), and multiparticle collision dynamics (MPC) that is also known as stochastic rotation dynamics (SRD). Dissipative particle dynamics was originally introduced by Hoogerbrugge and Koelman in 1992 [16]. This method combines molecular dynamics and Langevin dynamics. The system consists of a set of particles with continuous positions and velocities, whose time evolution is described by Newton's equations of motion. The force terms in DPD make it different from molecular dynamics. The force acting on a particle has three parts, each of which is a sum of pair forces. The three forces are a conservative force, a dissipative force and a random force. In traditional $\mathrm{DPD}$, the conservative force is chosen to be soft repulsive, which makes it posssible to use a large time step and achieve rapid equilibration. The dissipative force is the friction force that acts on the relative velocities of the particles. The random force compensates the loss of kinetic energy due to dissipative forces. Both dissipative and random forces together ensure that collision of particles is an isothermal process. 
The direct simulation Monte-Carlo (DSMC) method was proposed by Bird [17]. This algorithm is a stochastic particle-based method to solve nonlinear time-dependent Boltzmann equations. In DSMC simulations, the state of the system is given by positions and velocities of the particles. Like DPD, positions and velocities of the particles here are continuous variables. The geometry under consideration is divided into small cubical volumes called collision cells, which is a coarse-graining of the system. The algorithm consists of two steps: free-streaming and collisions. In the free-streaming step, particles are moved as if they do not interact, and their positions are updated based on their velocities. In the collision step, particles within the cells are randomly selected for collision partners. According to kinetic theory, the collision probability for hard spheres is directly proportional to their relative velocity. If the particles $i$ and $j$ are selected as candidates for the collision, they are accepted as collision partners if $\left|\mathbf{v}_{i}-\mathbf{v}_{j}\right|>R v_{r}^{\max }$ where $v_{r}^{\max }$ is the local maximum relative speed and $R$ is a random number chosen uniformly on the interval $[0,1)$. Since only the magnitude of the relative velocity between particles is used in determining the collision probability, particles that are moving away from each other may also collide. Imposing conservation of momentum and energy provides four of six equations to determine post-collision velocities. The remaining two conditions are selected randomly, assuming that the direction of the post-collision relative velocity is uniformly distributed on the unit sphere.

In this thesis, multiparticle collision dynamics (MPC) developed by Malevanets and Kapral [18] is used. This method shares many features with DSMC described above. An important difference between DSMC and MPC is the collision step. In the MPC collision step, the pre-collision velocity vector $\mathbf{v}_{i}^{\prime}$ of particle $i$ in collision cell $\xi$ is rotated by a randomly chosen rotation operator $\widehat{\omega}_{\xi}$ relative to the average cell velocity $\mathbf{V}_{\xi}^{\prime}$ to give the post-collision velocity

$$
\mathbf{v}_{i}^{\prime \prime}=\mathbf{V}_{\xi}^{\prime}+\widehat{\omega}_{\xi}\left(\mathbf{v}_{i}^{\prime}-\mathbf{V}_{\xi}^{\prime}\right) .
$$

Here $\widehat{\omega}_{\xi}$ is a stochastic rotation matrix that rotates the velocities by either $+\alpha$ or $-\alpha$ and varies both in time and from cell to cell. This way of performing collisions is much more numerically efficient than DSMC.

MPC dynamics has been used by Lamura and Gompper [19] to simulate two-dimensional fluid flow past a circular and square cylinder. Following this work, Allahyarov and Gompper have simulated three-dimensional flow past a sphere by MPC dynamics [20]. In this 
work, they have pointed out the limitations of the reservoir method, which is a pressuredriven method that contains a reservoir of particles, and used gravity-driven flow with periodic boundary conditions instead. It has been found that flow profiles generated by gravity-driven flow are much more stable than those from the reservoir method. Reid et. al have simulated flow around fish-like shapes using $M P C$ dynamics [21]. Simulation of a model microswimmer has been recently done by using $M P C$ dynamics [22]. Results of all these simulations have been found to be in good agreement with empirical data and the Navier-Stokes equations.

Flows through axisymmetric stenosis have been simulated by using the axisymmetric lattice Boltzmann method recently [23]. DPD has been applied to simulate flows through the constriction by Darias [24]. In this work the periodic boundary conditions are employed along the flow direction and particle wall interactions are modeled by using the bounce-back boundary condition. The density and velocity profiles have also been presented in this work, though it is not a detailed study.

Motivated by the works mentioned above, in this thesis $M P C$ dynamics is applied to simulate three-dimensional flows through geometries with and without local constrictions. The case of particle aggregation is not considered in this study, but as a first step towards it, $M P C$ dynamics is applied to simulate flows through cylindrical geometries and the results are compared with the incompressible Navier-Stokes equation. It is the first time since the development of $M P C$ dynamics, that it is applied to simulate steady flows through a three-dimensional axisymmetric constricted cylinder.

\subsection{Specific Aim And Methodology}

The specific aim of this thesis is to study dynamics of steady flows through an axisymmetric constricted cylinders with no-slip and slip boundary conditions using $M P C$. To implement these boundary conditions the particle interactions with the cylider wall are modeled by bounce-back $(B B)$ and loss in tangential $(L I T)$ boundary conditions.

In the $B B$ boundary condition, both tangential and normal components of particle velocities are reversed when particles hit the solid wall. In case of $L I T$, particles lose the tangential component of velocity after hitting the solid wall and the normal component of velocity is reversed. The first boundary condition is equivalent to macroscopic no-slip 
and the second one leads to slip. Interesting differences have been found in the velocity profiles obtained from these two boundary conditions. This is the first time $M P C$ dynamics has been applied to simulate steady flows through three-dimensional axisymmetric constricted cylinders. The constriction is represented by a smooth cosine shaped function [6] that can serve as an idealized model of vascular constrictions. The MPC dynamics simulates compressible flows. In order to be in the regime of incompressibility, the Mach number, which is defined as the ratio of the mean fluid speed to the speed of sound in a given fluid medium, should be much less than one. This means that the incoming fluid velocity must be low enough to satisfy this requirement. Increasing the Reynolds number without changing the mean fluid velocity would require larger system sizes, and hence more number of particles in the system, which amounts to requiring large computer memory and long simulation times. For these reasons simulations are performed at low values of the Reynolds number. The author would like to mention that it is not impossible to perfom simulations at high values of the Reynolds number using $M P C$ dynamics, though they are computationally demanding for the geometries considered in this work.

\subsection{Thesis Organization}

In Chapter two, the fundamental equations of incompressible fluid dynamics, namely Navier-Stokes and the continuity equation are discussed. The standard Poiseuille flow equation is derived from the Navier-Stokes equations under appropriate assumptions. Dynamic similarity and the Reynolds number are discussed as well. Next the numerical details of $M P C$ dynamics are discussed. $M P C$ dynamics consists of a multiparticle collision step followed by gravity- driven flow, thermostatting, free-streaming, and averaging to obtain the macroscopic flow field. Finally, finite element and $M P C$ simulation parameters,and flow geometry are discussed briefly. Chapter three contains the results followed by a summary of key findings and a discussion. Chapter four contains a summary, conclusions and future work. A dimensionalization scheme is explained in Appendix A. 


\section{Chapter 2}

\section{Fluid Dynamics}

\subsection{Chapter Overview}

In this chapter the basic equations of continuum fluid dynamics are discussed first and then the numerical implementation of $M P C$ dynamics is explained. The derivations of some of the standard fluid dynamics equations are skipped as they can be found in classic fluid dynamics textbooks [25]. Navier-Stokes equations for viscous incompressible fluids are discussed first. Then a case of steady flow of a Newtonian fluid through a cylindrical geometry is discussed and the concepts of the Reynolds number and dynamic similarity are introduced. Numerical implementation of $M P C$ dynamics is explained next. The $M P C$ dynamics is based on rigorous principles of statistical mechanics. It is not possible to cover all of these principles here, however some of those are briefly explained whenever necessary. The multiparticle collision step is explained, and then it is shown that the collisions conserve momentum and energy. The method of force driven flow using a Verlet algorithm and the Galilean invariant thermostat are introduced next. It is shown that collisions and free-streaming conserve the phase space volume, which is nothing but a Liouville theorem in classical statistical mechanics. The discussion then continues with a brief mention of the Boltzmann $H$ theorem. The boundary conditions to model particle wall interactions are discussed next. The cumulative averaging procedure used to make a connection with the solution of the Navier-Stokes equations, and expressions to compute the viscosity for $M P C$ fluid are discussed briefly. Finally the finite element simulation parameters and flow geometry are discussed briefly. 


\subsection{Continuum fluid dynamics}

Fluid dynamics is the theory of motion of liquids and gases. A typical fluid system consists of the billions of atoms or molecules. It is very difficult to keep track of each individual atom or molecule. Since fluid motion is a collective phenomenon, it is quite often really a waste of time to keep track of trajectories of individual atoms or molecules. In the continuum picture, the fluid can be thought of as made up of small volumes called fluid elements or fluid particles which themselves contain many atoms or molecules. The state of the fluid is determined by the density $\rho(\mathbf{r}, t)$, the velocity vector $\mathbf{v}(\mathbf{r}, t)$ and the pressure $p(\mathbf{r}, t)$, where $\mathbf{r}=(x, y, z)$ are the spatial coordinates and $t$ is the time. Please note that throughout the thesis, bold-faced quantities denote vectors.

The fundamental equations of motion in incompressible viscous fluid dynamics are Navier-Stokes equations (2.1). These equations are nothing but Newton's second law applied to the motion of a fluid element. The equation (2.2) is the continuity equation.

$$
\begin{gathered}
\rho\left(\frac{\partial \mathbf{v}}{\partial t}+(\mathbf{v} \cdot \nabla) \mathbf{v}\right)=-\nabla p+\mu \nabla^{2} \mathbf{v} \\
\rho \nabla \cdot \mathbf{v}=0 .
\end{gathered}
$$

In (2.1), the first term in the brackets is the usual acceleration term, and the second term represents convective acceleration that makes the entire equation non-linear. The convective acceleration is caused by the change in velocity over position. The first term on the right-hand side is the pressure gradient. The second term is the viscous force where $\mu$ is the dynamic viscosity of a given fluid, and is a measure of resistance. The CGS units of dynamic viscosity are "Poise" and the MKS unit is Pa.s. Poise is defined as $1 \mathrm{P}=1 \mathrm{~g} \cdot \mathrm{cm}^{-1} \cdot \mathrm{s}^{-1}$ and the relation to SI units is $1 \mathrm{P}=0.1 \mathrm{~Pa} \cdot \mathrm{s}$. Equations (2.1) and (2.2) are a system of four equations with four unknowns ( $\mathbf{v}$ and $p$ ) and can be solved numerically with appropriate boundary conditions.

There are very few situations where equations (2.1) and (2.2) can be solved analytically. The flows of viscous fluids can be very complex, ranging from laminar to turbulent flows. The principle of dynamic similarity and dimensional analysis can be used to construct the solutions of the Navier-Stokes equations. Generally, in fluid dynamics, one deals with flows through different geometries, or the flow past different geometrical ob- 
jects, that introduce a characterstic linear dimension that is often the diameter $D$ of the tube, or that of an object. Another important quantity in fluid dynamics is the kinematic viscosity, and is defined as $\nu=\frac{\mu}{\rho}$. The kinematic viscosity is measured in $\mathrm{m}^{2} / \mathrm{s}$ which are the units of a diffusion coefficient, and hence kinematic viscosity is also called momentum diffusivity. Let us consider a stationary flow with velocity scale $\mathrm{U}$, from these parameters, a dimensionless number called the Reynolds number can be defined as

$$
R e=\frac{D U}{\nu} .
$$

Using the dimensionless quantities $\frac{\mathbf{r}}{D}$ and $\frac{\mathbf{v}}{U}$ the solution of the steady Navier-Stokes equation can be written in the form

$$
\frac{\mathbf{v}}{U}=\mathbf{f}\left(\frac{\mathbf{r}}{D}, R e\right) .
$$

This holds for every flow with the same Reynolds number. Such flows are called dynamically similar flows, and the respective solutions can be simply obtained by rescaling. From equation (2.3), it can be seen that the Reynolds number is a ratio of inertial to viscous forces and equation (2.4) shows that the solution of the Navier-Stokes equations only depends on this dimensionless number.

A flow of fluid through a pipe of uniform cross-section is known as Hagen-Poiseuille flow. The velocity profile of Hagen-Poiseuille flow can be derived by using the NavierStokes equation in a cylindrical coordinate system under the following assumptions:

- The flow is steady.

- Radial and azimuthal components of fluid velocity are zero.

- Flow is axisymmetric and fully developed.

With these assumptions, the Navier-Stokes equations become

$$
\frac{1}{r} \frac{\partial}{\partial r}\left(r \frac{\partial v_{y}}{\partial r}\right)=\frac{1}{\mu} \frac{\partial p}{\partial y},
$$

where $v_{y}$ is the only non-vanishing velocity component along the axial $y$-direction. The 
solution of $(2.5)$ can be written as

$$
v_{y}=\frac{1}{4 \mu} r^{2} \frac{\partial p}{\partial y}+A \ln r+B
$$

where $A$ and $B$ are constants. Since the velocity should be finite at $r=0, A=0$ and imposing no-slip boundary conditions at the walls, which requires $v_{y}=0$ at $r=R$, where $R$ is the radius of the pipe gives,

$$
B=-\frac{1}{4 \mu} \frac{\partial p}{\partial y} R^{2}
$$

Putting the values of the constants in equation (2.7), we get the famous parabolic velocity profile,

$$
v_{y}=-\frac{1}{4 \mu} \frac{\partial p}{\partial y}\left(R^{2}-r^{2}\right)
$$

Note that the maximum velocity occurs at the centre, and has the value

$$
v_{y}^{\max }=-\frac{R^{2}}{4 \mu} \frac{\partial p}{\partial y}
$$

and is commonly referred to as the centerline velocity $U_{c}$. Note that in these equations, the pressure gradient is typically imposed as a driving force of the fluid, and hence is known. The pressure $p$ can then be found by simple integration.

In the subsequent simulations, the centerline velocity is changed by changing the pressure gradient, and it is also used as velocity scale to determine the Reynolds number.

\section{$2.3 \quad M P C$ dynamics}

\subsubsection{Multiparticle collision}

The system is made up of $N$ identical point particles of unit mass $m$ that are uniformly distributed over cells on a regular lattice. There are $L_{x}, L_{y}$ and $L_{z}$ cells in the $x$, $y$ and $z$-directions respectively, and each cell $\xi$ contains $n$ particles on average. The continuous positions $\mathbf{r}_{i}$ and velocities $\mathbf{v}_{i}$ of the particles $(i=1,2, \ldots, N)$ are updated at discrete time intervals $\Delta t$. The particle positions and momenta together form a phase 
space that is denoted by $\Gamma=\left(\mathbf{r}_{i}^{N}, \mathbf{p}_{i}^{N}\right)=\left(\mathbf{r}_{1}, m \mathbf{v}_{1}, \ldots, \mathbf{r}_{N}, m \mathbf{v}_{N}\right)$. There are $N$ particles in the system, each particle has three position and three momentum coordinates and hence the dimension of phase space is $6 N$. The $M P C$ algorithm in this work consists of a numerically efficient mass, momentum and energy-conserving collision rule $[1,18]$ followed by acceleration, thermostatting and free-streaming. In the collision rule, the pre-collision velocity vector $\mathbf{v}_{i}^{\prime}$ of particle $i$ is rotated by a randomly chosen rotation operator $\widehat{\omega}_{\xi}$ relative to the average cell velocity (or center of mass velocity) $\mathbf{V}_{\xi}^{\prime}$ to give the post-collision velocity

$$
\mathbf{v}_{i}^{\prime \prime}=\mathbf{V}_{\xi}^{\prime}+\widehat{\omega}_{\xi}\left(\mathbf{v}_{i}^{\prime}-\mathbf{V}_{\xi}^{\prime}\right)
$$

Here $\widehat{\omega}_{\xi}$ is a stochastic rotation operator that rotates the velocities by either $+\alpha$ or $-\alpha$ and varies both in time and from cell to cell. The rotations here are not performed by using the Euler angles as it is difficult numerically, and can induce errors as well. Instead quaternions [1] have been used here to perform rotations. The quaternions are non-commutative extensions of complex numbers, sometimes also called hypercomplex numbers.

It can also be shown that the above transformation conserves momentum and energy. The proof is as follows: multiplying (2.10) by $m$ and summing over the number of particles $N_{\xi}$ in a given cell, and using the fact that the rotation operator is the same for all the particles in a given cell, gives

$$
\begin{gathered}
\sum_{i=1}^{N_{\xi}} m \mathbf{v}_{i}^{\prime \prime}=\sum_{i=1}^{N_{\xi}} m\left(\mathbf{V}_{\xi}^{\prime}+\widehat{\omega}_{\xi}\left(\mathbf{v}_{i}^{\prime}-\mathbf{V}_{\xi}^{\prime}\right)\right)=\sum_{i=1}^{N_{\xi}} m \mathbf{v}_{i}^{\prime} . \\
\sum_{i=1}^{N_{\xi}} \frac{m}{2}\left|\mathbf{v}_{i}^{\prime \prime}\right|^{2}=\sum_{i=1}^{N_{\xi}} \frac{m}{2}\left|\mathbf{V}_{\xi}^{\prime}+\widehat{\omega}_{\xi}\left(\mathbf{v}_{i}^{\prime}-\mathbf{V}_{\xi}^{\prime}\right)\right|^{2}=\sum_{i=1}^{N_{\xi}} \frac{m}{2}\left|\mathbf{v}_{i}^{\prime}\right|^{2}
\end{gathered}
$$

Figure 2.1 shows the diagrammatic representation of a collision for two particles in two dimensions as a demonstration of the rule, although the rule works for any number of particles in a given collision cell. Note that in the diagram, the $\xi$ subscript has been dropped for clarity. Note that the collisions in MPC dynamics are not pairwise collisions as used in DSMC, and hence much more efficient numerically. 

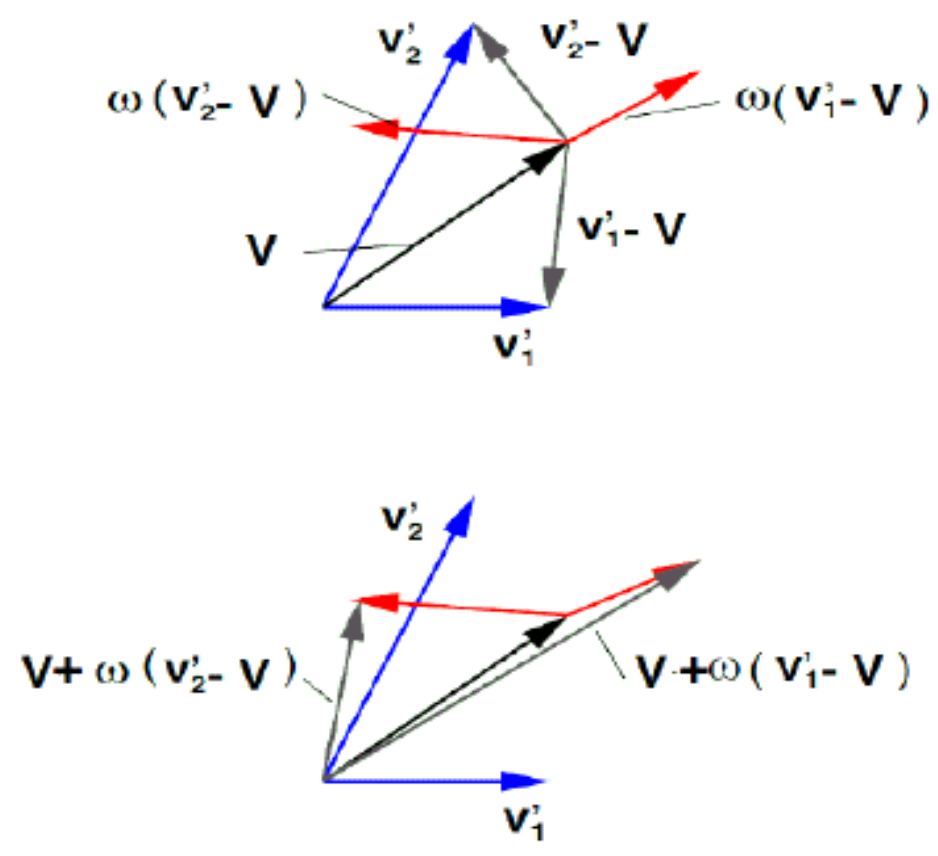

Figure 2.1: Diagrammatic representation of the multiparticle collision rule. The upper pannel shows the center of mass velocity and the pre-collision velocities of two particles relative to the center of mass. The lower pannel shows the result of adding back the center of mass velocities to get post-collision velocities. The figure is adapted from Anatoly Malevanets and Raymond Kapral [1] 


\subsubsection{Gravity-Driven Flow and Thermostatting}

The direction of flow in section (2.2) was $z$ direction and in $M P C$ simulations it is along $y$ direction. To create flow, a constant force $\mathbf{F}=m \mathbf{g}=(0, m g, 0)$ is applied along the $y$-direction to all the fluid particles and the periodic boundary conditions are imposed along the $y$ direction. The post-collision velocities of the particles are then updated by means of a Verlet algorithm according to

$$
\mathbf{v}_{i}^{\prime \prime \prime}=\mathbf{v}_{i}^{\prime \prime}+\frac{\mathbf{F}_{i}}{m} \Delta t
$$

When an external force is applied to the system this way, energy is pumped into the system and consequently the actual temperature, $T^{\prime}$, of the system increases. For isothermal flow at temperature $T$, the system has to be coupled to a thermostat. In this work a velocity profile unbiased Galilean invariant thermostat $[26,27]$ is used to achieve isothermal flow conditions. The thermostat relates the instantaneous local temperature in a cell to the mean square deviation of the particle velocities computed from the center of mass velocity of that cell. Mathematically, thermostatting scales the velocities according to

$$
\begin{gathered}
k_{B} T^{\prime}=\frac{m}{N_{\text {free }}} \sum_{\xi} \sum_{i \epsilon \xi}\left(\mathbf{v}_{i}^{\prime \prime \prime}-\mathbf{V}_{\xi}^{\prime \prime \prime}\right)^{2}, \\
N_{\text {free }}=\sum_{\xi} \begin{cases}3\left(N_{\xi}-1\right) & \left(N_{\xi}>1\right), \\
0 & \left(N_{\xi} \leq 1\right) .\end{cases} \\
\mathbf{v}_{i}=\mathbf{V}_{\xi}^{\prime \prime \prime}+\sqrt{\frac{T}{T^{\prime}}}\left(\mathbf{v}_{i}^{\prime \prime \prime}-\mathbf{V}_{\xi}^{\prime \prime \prime}\right)
\end{gathered}
$$

In equations (2.14) and (2.15), recall that $N_{\xi}$ is the number of particles in cell $\xi$. Note that the local temperature is not defined in cells that have one or no particles. The thermostat acts by rescaling the relative velocities $\left(\mathbf{v}_{i}^{\prime \prime \prime}-\mathbf{V}_{\xi}^{\prime \prime \prime}\right)$ by $\sqrt{\frac{T}{T^{\prime}}}$ at each time step according to equation (2.16). 


\subsubsection{Free-streaming, Liouville and $H$-Theorems}

Free-streaming updates the positions of particles according to

$$
\mathbf{r}_{i}=\mathbf{r}_{i}^{\prime}+\mathbf{v}_{i} \Delta t
$$

where $\mathbf{r}_{i}^{\prime}$ is the position of particle $i$ at the beginning of the time step and $\mathbf{v}_{i}$ is the velocity after collision, acceleration and thermostatting have taken place. It can be verified that the transformation corresponding to free-streaming has unit Jacobian determinant and hence the transformation is canonical.

It can be shown that the collision and free-streaming preserves the phase space volume, which is nothing but a Liouville theorem. Let the phase point of the system at time $t$ and $t+\Delta t$ be $\left(\mathbf{r}_{i}^{\prime}{ }^{N}, \mathbf{v}_{i}^{\prime}{ }^{N}\right)$ and $\left(\mathbf{r}_{i}{ }^{N}, \mathbf{v}_{i}{ }^{N}\right)$. The phase space volume at time $t+\Delta t$ can be written as,

$$
d \mathbf{x}^{N}=d \mathbf{r}^{N} d \mathbf{v}^{N} .
$$

Now let $p\left(\widehat{\omega} \mid \mathbf{v}^{\prime N}\right)$ be the conditional probability of rotation given the velocity. Using this, equation (2.18) can be written as

$$
d \mathbf{x}^{N}=d \mathbf{r}^{N} \sum_{\mathbf{v}^{\prime N}} p\left(\widehat{\omega} \mid \mathbf{v}^{\prime N}\right) d \mathbf{v}^{\prime N}=d \mathbf{x}^{\prime N} \sum_{\mathbf{v}^{\prime N}} p\left(\widehat{\omega} \mid \mathbf{v}^{\prime N}\right)=d \mathbf{x}^{\prime N},
$$

where $d \mathbf{r}^{N}=d \mathbf{r}^{\prime N}$ and $\sum_{\mathbf{v}^{\prime N}} p\left(\widehat{\omega} \mid \mathbf{v}^{\prime N}\right)=1$ which follows from the principle of semidetailed balance and the fact that velocities do not depend on the rotations. Finally it can be shown [1] that the MPC dynamics algorithm satisfies a Boltzmann $H$-theorem if the collision rule satisfies the principle of semi-detailed balance, and if the assumption of molecular chaos is valid. The Boltzmann $H$-functional has the form,

$$
H=\int d \mathbf{v} d \mathbf{r} f(\mathbf{v}, \mathbf{r}, t) \ln f(\mathbf{v}, \mathbf{r}, t),
$$

where $f(\mathbf{v}, \mathbf{r}, t)$ is the reduced single particle distribution function. The $H$-functional decreases in time and attains the minimum value for the equilibrium Maxwell-Boltzmann distribution,

$$
f(\mathbf{v}, \mathbf{r}, t)=\frac{N}{V}\left(\frac{m}{2 \pi k_{B} T}\right)^{\frac{d}{2}} e^{-m\|\mathbf{v}-\mathbf{V}\|^{2} / 2 k_{B} T},
$$


where $N$ is the average number of particles in the system, $V$ is the system volume, $\mathbf{V}$ is the average velocity of particles, $k_{B}$ is the Boltzmann constant, and $T$ is the system temperature. The $H$-theorem guarantees that the dynamical system relaxes to the thermodynamically correct Gibbs equilibrium state. Please note that the other popular particle-based methods, such as lattice Boltzmann and lattice gas, exhibit numerical instabilities because of nonmonotonic decay of the $H$-functional.

It has also been shown that the hydrodynamic equations can be derived from the reduced probability distribution by invoking a Chapman-Enskog expansion [18]. The second-order terms in the Chapman-Enskog expansion give the familiar Navier-Stokes equations after averaging.

\subsubsection{Boundary conditions}

The dynamics of fluid flows is strongly affected by the presence of interfaces. On the macroscopic scale, for the fluids in contact with a solid phase, the normal component of velocity at the interface must be zero. This represents an impenetrable boundary. The tangential velocity inherently depends upon the molecular interactions at the interface. There are two ideal situations: no-slip (or stick) and slip boundary conditions. In case of no-slip, the tangential velocity of the fluid relative to that of the boundary vanishes. In case of slip, the tangential component of the fluid velocity is non-zero.

There are several ways of implementing these boundary conditions at the mesoscopic level. In this work, two boundary conditions, namely the bounce-back (BB) and loss of tangential and reversal of normal (LIT) are used. The BB boundary condition was first proposed for $M P C$ dynamics by Malevanets and Kapral [1, 18]. This boundary condition is an analog of no-slip boundary conditions used in lattice Boltzmann simulations [23]. In $B B$, the normal and tangential components of particle velocities are reversed after the collision with the wall. As a result, the average relative velocity of the fluid near the wall is zero since the relative velocity distribution of particles reflected from the wall mirrors the distribution of particles approaching the wall [28]. This case corresponds to a perfectly rough wall (or frictional wall). In case of $L I T$, the tangential component of the particle velocity is lost and the normal component is reversed. As a result, the average fluid velocity near the wall is non-zero which results in slip. Such type of wall is called a rough wall which is different from a perfectly rough wall. Since blood vessel walls are 
not very smooth because of endothelium and other layers and porous in nature, a small slip may be present at the wall. Instead of a perfectly rough wall, one may regard the vessel wall as a rough wall. The boundary conditions in particle-based simulations can be written in a more general mathematical form

$$
\begin{gathered}
\mathbf{v}_{n}=-\mathbf{v}_{n}^{\prime}, \\
\mathbf{v}_{t}=(2 \lambda-1) \mathbf{v}_{t}^{\prime} .
\end{gathered}
$$

The subscripts $n$ and $t$ refer to normal and tangential components and $\mathbf{v}, \mathbf{v}^{\prime}$ represent post and pre-collision velocities of an individual particle hitting the wall. From the above equations, it can be seen that $\lambda=0$ corresponds to $B B$, and $\lambda=0.5$ corresponds to LIT. Note that the degree of slip can be varied by changing values of $(2 \lambda-1)$. The perfect slip can be achieved by $\lambda=1$ and would lead to a uniform flow. Please note that the LIT is a dissipative boundary condition and does not conserve energy. Since a thermostat is employed in these simulations, this is not an issue.

\subsubsection{Grid-shifting}

If the system temperature and hence the average distance travelled by a particle between two successive collisions (eg. the mean free path) are low, that is if the mean free path is less than the cell size, then the same particles could collide more than once with each other. Multiple collisions can also happen near boundaries in the flow domain. This would break Galilean invariance, violating the molecular chaos assumption. To solve this problem, the grid shifting mechanism proposed by Ihle and Kroll [29, 30] is employed. In the grid shifting, all particles are shifted by the same uniformly distributed random translation vector before the collision step. The shift randomizes the positions of the particles in a cell and provides an isotropic collision environment so that Galilean invariance is restored.

\subsection{Averaging Procedure}

In particle-based simulations of fluid flow, computation of mean fluid velocity is very easy but can induce measurement bias as has been explained by Tysanner and Garcia [31]. 
The two commonly used averaging methods are known as sample average measurement (SAM) and cumulative average measurement (CAM). The SAM obtains the average particle velocity in each cell at each sample, and then averages these over all the samples. The CAM sums the velocities of all particles in a cell over all the samples, and then divides this cumulative total by the cumulative total number of particles in the cell to get the mean velocity per cell. It has been shown in reference [31] that SAM can have bias due to the correlations of fluctuations in the system. To avoid these correlations CAM is adopted in this work. CAM can be mathematically described as,

$$
\left\langle\mathbf{u}_{\xi}\right\rangle=\frac{\sum_{j=1}^{S} \sum_{i \xi \xi}^{N_{\xi}\left(t_{j}\right)} \mathbf{v}_{i}\left(t_{j}\right)}{\sum_{j=1}^{S} N_{\xi}\left(t_{j}\right)} .
$$

In the above equation $\xi$ again refers to the cell number, $i \epsilon \xi$ means that particle $i$ is inside cell number $\xi$ at time $t_{j}, N_{\xi}\left(t_{j}\right)$ is the number of particles in cell $\xi$ at time $t_{j}$ and $\left\langle\mathbf{u}_{\xi}\right\rangle$ is the macroscopic velocity vector for the particles in the same cell. In addition, $S$ represents the number of samples used in the averaging process. Rather than using different samples for fixed time intervals, we assume ergodicity and use one very long simulation instead. Computing averages from one simulation is common $[1,18,19]$ and preliminary tests for a slightly smaller geometry using five different initial conditions showed no changes in the observed features in the results reported in this thesis. It has been argued that the stochastic nature of multiparticle collision dynamics leads to noisy averages [27]. The averages obtained by the CAM method are smooth. The latter is further evidenced in the surface velocity plots and velocity profiles shown in Chapter three.

\subsection{Transport Coefficients}

The beauty of the MPC dynamics is that analytic calculation of viscosity and other transport coefficients is possible. It has been shown by Noguchi and Gompper[32] that the fluid viscosity $\mu$ consists of the sum of a kinetic component $\mu_{k i n}$ and a collisional part

$\mu_{\text {coll }}$. For the $\alpha=\frac{\pi}{2}$ rule (which is the rule used in this work), the simple 3-d expressions are given by 


$$
\begin{gathered}
\mu_{\text {kin }}=\left(\frac{\rho k_{B} T}{m}\right) \Delta t\left[\frac{5 n}{6\left(n-1+e^{-n}\right)}-\frac{1}{2}\right], \\
\mu_{\text {coll }}=\frac{m}{18 a_{0} \Delta t}\left(n-1+e^{-n}\right), \\
\mu=\mu_{\text {kin }}+\mu_{\text {coll }}
\end{gathered}
$$

where $n$ is the average number of particles in a cell, $a_{0}$ is the length of the $M P C$ cell and $\rho=m n / a_{0}{ }^{3}$ is the mass density. In an unconstricted tube, the constant force corresponds to a pressure drop per unit length of $d P / d y=-\rho m g$, where $d P / d y$ is the pressure gradient in our flow geometry. When the gravitational force balances the shear force, the system becomes stable and the velocity profile settles to the laminar Poiseuille flow, equation (2.8) or in the flow geometry used, (see Figure 2.2),

$$
v_{y}(r)=\frac{1}{4 \mu} \frac{d P}{d y}\left(r^{2}-R^{2}\right),
$$

where $v_{y}(r)$ is the non-vanishing component of the macroscopic flow velocity which depends only on the radial distance $r$ from the $y$-axis, and $\mu$ is the dynamic viscosity computed from (2.27). Note that in the unconstricted geometry, the $y$-component of the average velocity of (2.24) should agree with that of (2.28), and the $x$ and $z$ components should vanish.

To vary the Reynolds number in the simulations, the centerline velocity $U_{c}$ can be obtained from (2.28) giving,

$$
U_{c}=-\frac{d P}{d y} \frac{D^{2}}{16 \mu}=\frac{\rho m g D^{2}}{16 \mu} .
$$

Thus, by varying the value of $g$, the Reynolds number

$$
R e=\frac{\rho U_{c} D}{\mu}
$$

is changed in the subsequent simulations. 


\subsection{Finite element simulation}

Two-dimensional axisymmetric steady-state Navier-Stokes equations are solved using the finite element package called Comsol Multiphysics (Comsol Inc., Burlington, MA, USA). The direction of flow in these simulations is along the $y$ axis. The density and temperature of the fluid are assumed to be uniform in the flow field and the viscosity of the fluid is constant. The walls are assumed to be rigid with no-slip boundary conditions. The inlet boundary condition is fully developed Poiseuille flow, and at the outlet, boundary condition is pressure with no viscous stress. The simulations in Comsol Multiphysics are for realistic dimensionful blood flow parameters, and using the non-dimensionalization explained in an Appendix allows to compare the Comsol Multiphysics results with the MPC simulations.

The density of the fluid is taken to be $\rho=1000 \mathrm{~kg} / \mathrm{m}^{3}$, the dynamic viscosity $\mu=$ $1.238 X 10^{-3}$ Pa.s, the diameter $D=4 \mathrm{~mm}$, and the centerline velocity $U_{c}=2.18811 \mathrm{~mm} / \mathrm{s}$ which gives $R e \approx 7$. The geometry of the stenosis was generated using MATLAB, and then imported into Comsol multiphysics to generate the finite element mesh using Lagrange $P_{2} P_{1}$ elements. The resulting linear system of equations is then solved using the parallel direct sparse solver interface (PARDISO). Number of degrees of freedom used were 549336, number of elements were 120904 and minimum element quality was 0.608. For further details on this, please refer to the Comsol Multiphysics Modeling guide [33].

\subsection{Geometry and $M P C$ Parameters}

The flow geometry is shown in Figure 2.2. The radius of the cylinder, $R(y)$, is a function of the longitudinal coordinate $y$, with cross-section in the $x z$-plane. Following Varghese, Frankel and Fischer [6], the geometry as chosen,

$$
\begin{gathered}
R(y)=\frac{D}{2}\left[1-s_{c}\left(1+\cos \left(\frac{4 \pi\left(y-y_{c}\right)}{D}\right)\right)\right] \\
x=R(y) \cos \theta \\
z=R(y) \sin \theta
\end{gathered}
$$




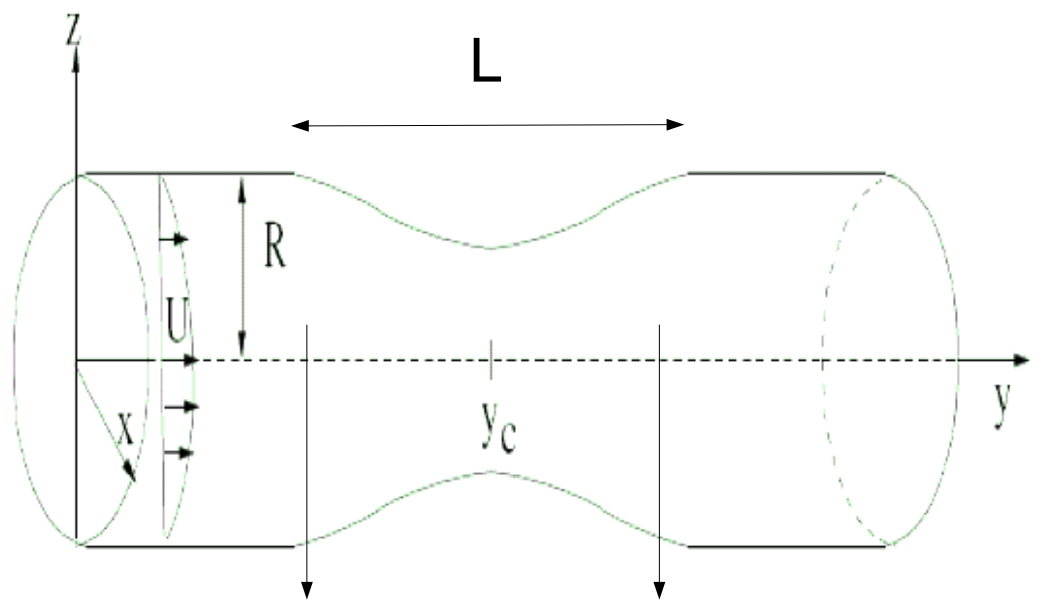

A $\quad$ B

Figure 2.2: Flow Geometry

\begin{tabular}{|c|c|c|}
\hline Parameter & Symbol & value \\
\hline \hline Temperature & $k_{B} T$ & 1.0 \\
\hline Lattice cell size & $a_{0}$ & 1.0 \\
\hline rotational angle & $\alpha$ & $\pi / 2$ \\
\hline particle mass & $m$ & 1.0 \\
\hline Average particles per cell & $n$ & 15.0 \\
\hline time step & $\Delta t$ & 1.0 \\
\hline Number of Cells along $X$ direction & $L_{x}$ & 17 \\
\hline Number of Cells along $Y$ direction & $L_{y}$ & 1200 \\
\hline Number of Cells along $Z$ direction & $L_{z}$ & 17 \\
\hline Center of the stenosis & $y_{c}$ & 226.5 \\
\hline
\end{tabular}

Table 2.1: Simulation Parameters 
where $D$ is the diameter of the unconstricted section, $y_{c}$ is the location of the center of the stenosis, the length of the stenosis is taken to be equal to $D / 2$, and the value for $s_{c}$ controls the severity or degree of the constriction. The degree of constriction is defined as the change in diameter per unit original diameter. For a $40 \%$ constriction, $s_{c}=0.2$. The cross- section velocity profiles, radial velocity profiles and the cross section density profiles presented in this work correspond to axial locations $y=222.5$ (upstream of $y_{c}=226.5$ ) and $y=229.5$ (downstream of $y_{c}=226.5$ ) for a cylinder of length 1200. These points are labeled in Figure 2.2 as A and B. The geometry is imposed on a rectangular grid and in the unconstricted portion there are 17 collision cells along $X$ and $Z$ directions, refer to Table 2.1. The centerline velocity in $M P C$ simulations is denoted by $\left.<\mathbf{u}_{\xi}(\mathbf{0})\right\rangle_{\mathbf{y}}=<\mathbf{u}_{\xi}(0, y, 0)>$ and in Comsol Multi-physics simulations, it is denoted by $v(r=0$ ), where $r=0$ represents the $y$ axis (an axis of symmetry). Both of these are scaled by $U_{c}$ defined earlier. In the cross-section velocity profiles, $\left\langle\mathbf{u}_{\xi}\right\rangle_{y}$ represents the $y$ component of the averaged velocity. In the radial velocity plots, $\left\langle\mathbf{u}_{\xi}\right\rangle_{z}$ represents the $z$ component of the averaged velocity. In the centerline density plots, $n^{*}$ represents number of particles along the centerline scaled by average number of particles $n$ in the $M P C$ cell.

In these simulations, there are a number of parameters whose values remain fixed and they are listed in the table 2.1. The rotation angle $\alpha=\frac{\pi}{2}$ is used, since it is computationally very fast and the value of the kinematic viscosity is the lowest, maximizing the Reynolds number [20]. All parameters are in nondimensional form and the consistent dimensionalization procedure is explained in an Appendix. In this work the case of 40 percent constriction with Reynolds numbers 4, 7 and 15 were considered for LIT and $B B$. Next, the Reynolds number was fixed $(R e=7)$ and cases of 30 and 60 percent constrictions were considered for LIT only. The Mach numbers in these simulations were $0.098,0.27$ and 0.42 and they can be computed using (2.34)

$$
M a=\frac{U_{c}}{\sqrt{\frac{3 k_{B} T}{m}}} .
$$




\section{Chapter 3}

\section{Results and Discussion}

\subsection{Poiseuille flow}

To test the method, simulations of steady flow through an unconstricted cylinder $\left(s_{c}=0\right.$, $R e \approx 7, g=0.005)$ are performed using the $M P C$ algorithm. The gravity-driven method as explained in the Section 2.3.2 was used to drive flow. The known case of Poiseuille flow was recovered successfully using $M P C$.

To further test the numerical implementation, the system was started from rest in the unconstricted cylinder and let to evolve to the parabolic profile described by equation (2.28). At rest means placing the particles uniformly in the flow domain, assigning velocities from a Maxwell-Boltzmann distribution (2.21) with mean zero at a temperature $T$. The simulation for $g=0.005(R e=7)$ showed that 8000 time steps were required to reach steady-state.

To speed up the simulations, an initial condition is used, in which the velocity in the $y$-direction is assigned according to (2.28). It was found that steady-state was reached after 3000 time-steps without affecting the final velocity profile. The Figure-3.1 shows the velocity profiles for a cylinder without constriction. Note that with $g=0.005$, $\rho=15, D=L_{z}=17$ and $\mu=6.668$ ( computed from (2.27)), the theoretically predicted centerline velocity using equation $(2.28)$ is $U_{c}=0.20$, but the centerline velocities that are actually observed in these simulations $\left(U_{c}=0.192\right)$ are 4 percent less than the theoretically predicted values.

The Reynolds number calculated using the theoretical value of centerline velocity is 


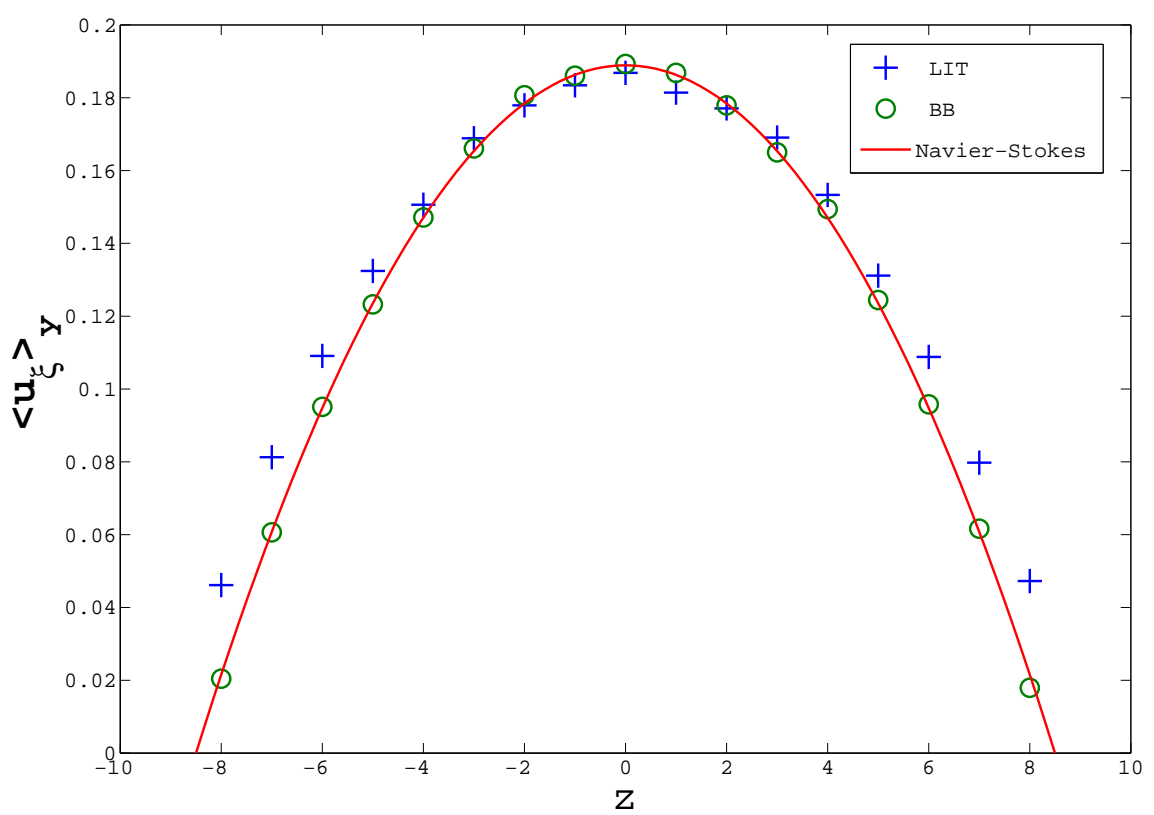

Figure 3.1: Cross-section velocity profiles for Poiseuille flow with $\mathrm{Re}=7$

7.7 and the one based on the observed centerline velocity is 7.4. In subsequent $M P C$ simulations, the Reynolds numbers were computed based on the observed centerline velocity in the unconstricted portion of the cylinder and then the same Reynolds numbers are used in Comsol Multiphysics (described in section 2.6) so that the two simulations have the same Reynolds numbers. Figure 3.1 shows three velocity profiles $L I T, B B$, and Navier-Stokes with no slip. The slip can be seen in the case of LIT, and the BB data points are in good agreement with Navier-Stokes (no-slip). It is worth noting that the cumulative averaging procedure used in this work gives better no-slip without using any artificial trick such as filling boundary cells by virtual particles [19].

The Figure 3.2 shows contour velocity plots for Poiseuille flow simulations for LIT and $B B$ simulations. 


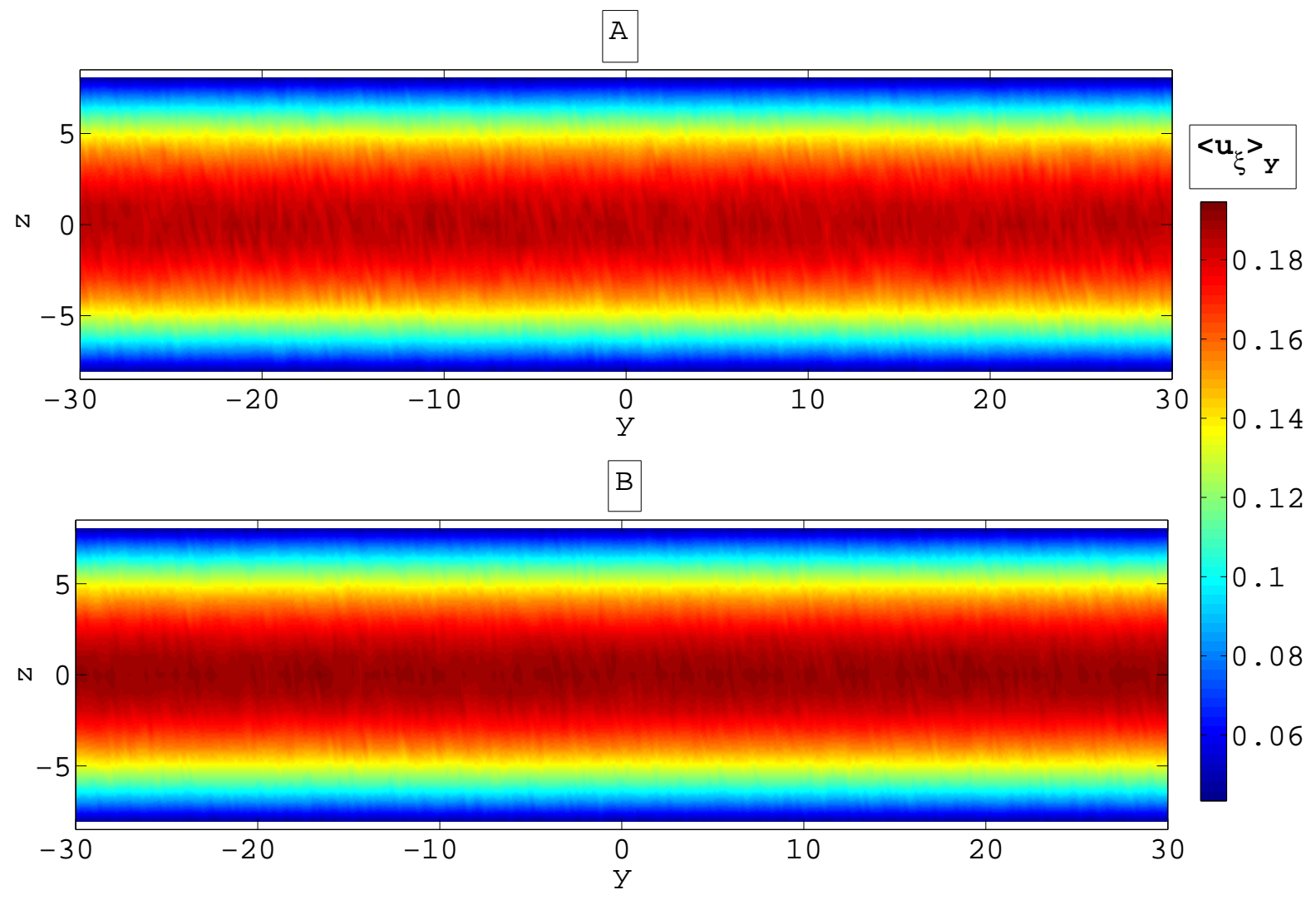

Figure 3.2: Contour velocity plots for Poiseuille flow using $M P C$ with $\mathrm{Re}=7$ using (A) $L I T$ and (B) $B B$ boundary conditions. 


\section{$3.2 \quad R e \approx 4, g=0.0025, s_{c}=0.2$}

Figure 3.3 shows the scaled centerline velocity profiles as a function of scaled axial coordinate. The figure shows that $B B$ and Navier-Stokes are in fairly good agreement. It can be seen in Figure 3.3 that after passing through the constriction, the centerline velocity in case of LIT shows a larger decrease as compared to $B B$ and Navier-Stokes. This is referred as a dip in subsequent discussions. The Figure 3.4 shows the cross-section velocity profiles upstream of the stenosis at A(see Figure 2.2). The Figure 3.4 shows, negative velocities near wall in case of the LIT. The maximum standard deviations for all the data points in the velocity profiles were found to be much less than $1 \%$. The standard deviations were determined by computing the ratio of root mean squared deviations of average velocity divided by the square root of the sample size. Note that there is a slight asymmetry in the LIT data near the center region (see Figure 3.4) which is likely a consequence of small fluctuations in the system at this low Reynolds number. Please note that $M P C$ describes fluctuating hydrodynamics. Figure 3.5 shows the cross section velocity profiles downstream of the constriction at location B. Figure 3.5 shows some distinct features in the case of LIT, where the velocity profile is flat near the wall and shows slip.

\subsection{Re $\approx 7, g=0.005, s_{c}=0.2$}

The Reynolds number is increased further by increasing the value of $g$. Figure 3.6 shows the scaled centerline velocity profiles as a function of scaled axial coordinate. It can be seen that the centerline velocities drop upstream of the constriction in case of both $L I T$ and $B B$. This could be a weak compressibility effect since the constriction can cause density variations. The centerline velocity profile in case of LIT again shows a dip in the post-constriction region. Figure 3.7 shows the cross-section velocity profiles upstream of the constriction, where negative velocities can be seen near the wall in the case of LIT. The Figure 3.8 shows the velocity profiles downstream of the constriction at B. The slip velocity can be seen in the case of LIT and is slightly higher than for $R e=4$. Figure 3.9 shows the cross-section density profiles upstream and downstream of the constriction at $\mathrm{A}$ and $\mathrm{B}$ respectively. The $B B$ gives more stable density profiles along the cross-section as compared to LIT. The radial velocity plots are also presented in Figures 3.10 and 


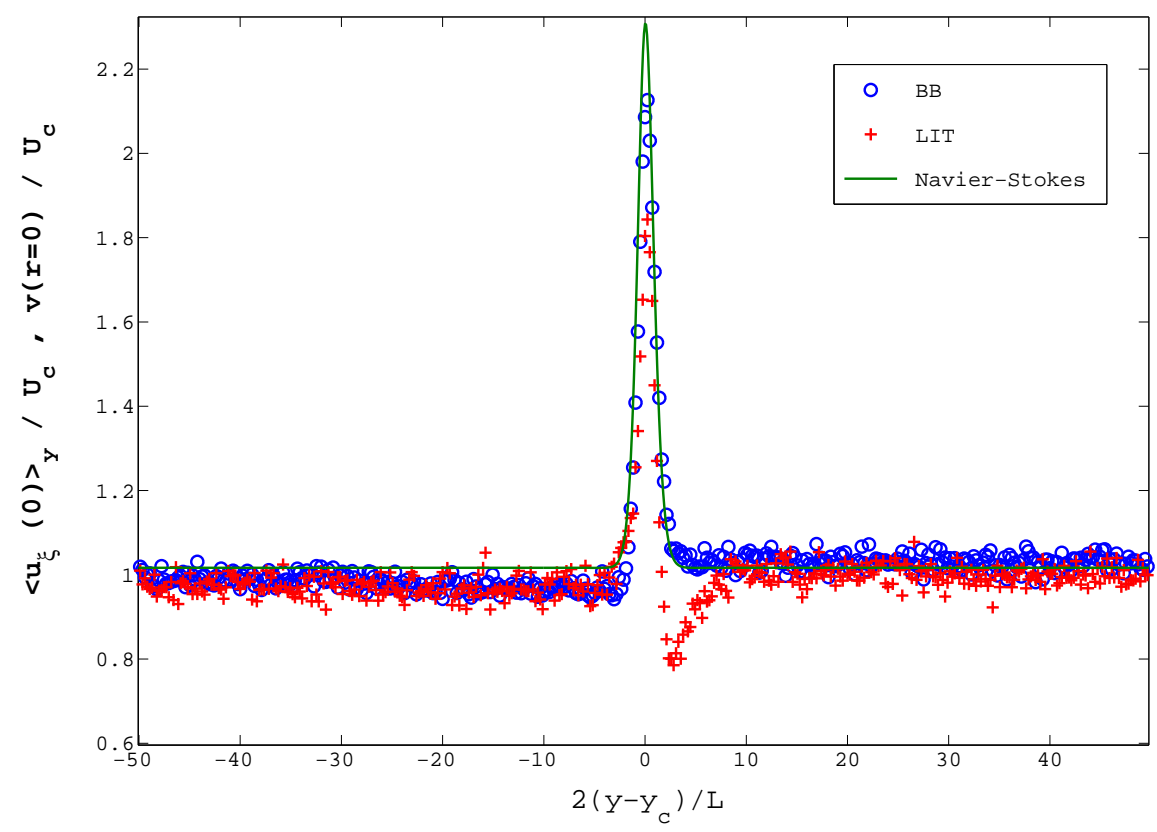

Figure 3.3: Centerline velocity profiles for 40 percent stenosis with $\operatorname{Re}=4$

3.11. The radial profiles in case of $B B$ are in good agreement to that of Navier-Stokes equations. The radial flow profiles for $R e=4$ and $R e=15$ are not presented here as they are similar to the one shown. In case of the cylinder without constriction, there is no radial flow.

\section{$3.4 R e \approx 15, g=0.01, s_{c}=0.2$}

The Reynolds number is increased to 15. Figure 3.12 shows the scaled centerline velocity profiles as a function of scaled axial coordinate. Compared to $R e=4$ and $R e=7$, a larger drop in centerline velocities upstream of the constriction can be seen in case of $L I T$ and $B B$, and the dip in the centerline velocity profile in the post-constriction region can be seen in case of LIT. Figure 3.13 shows the cross section velocity profiles upstream of the constriction. It can be seen that the velocities are no longer negative for this larger 


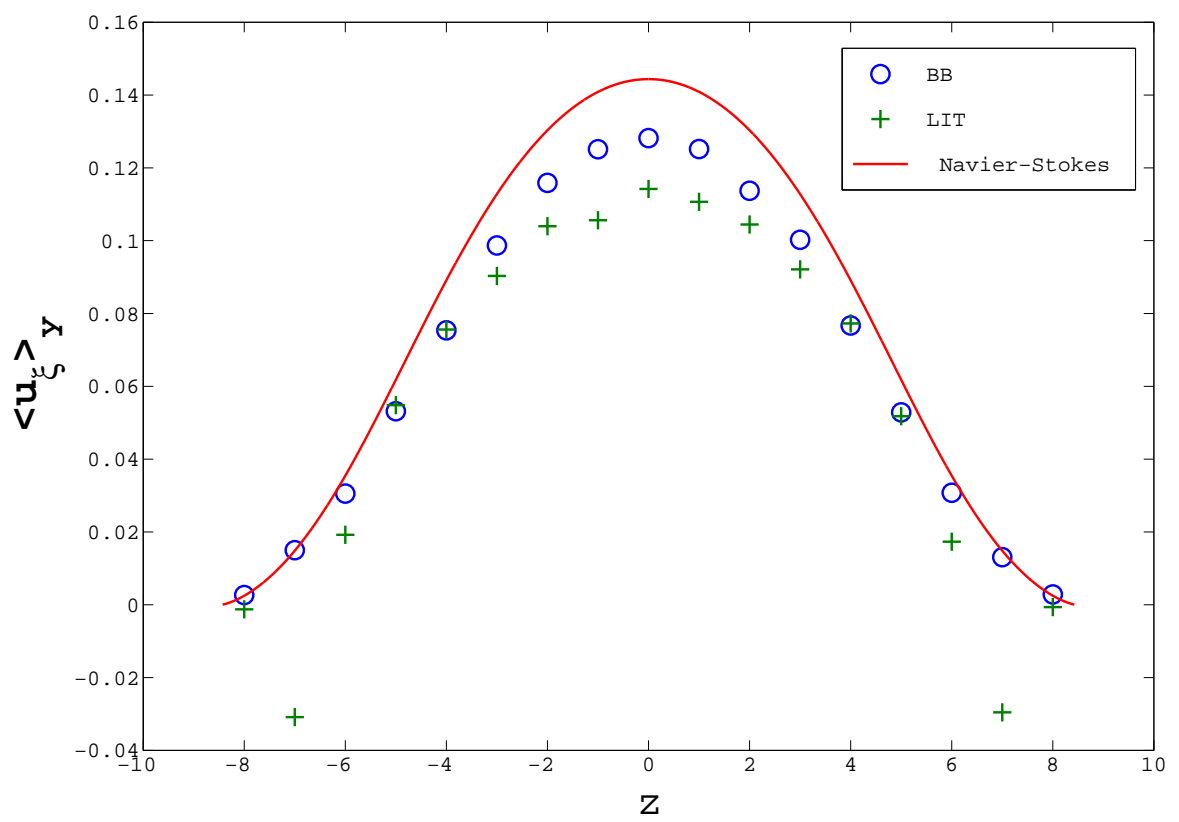

Figure 3.4: Cross-section velocity profiles upstream of the 40 percent stenosis at A with $\operatorname{Re}=4$ 


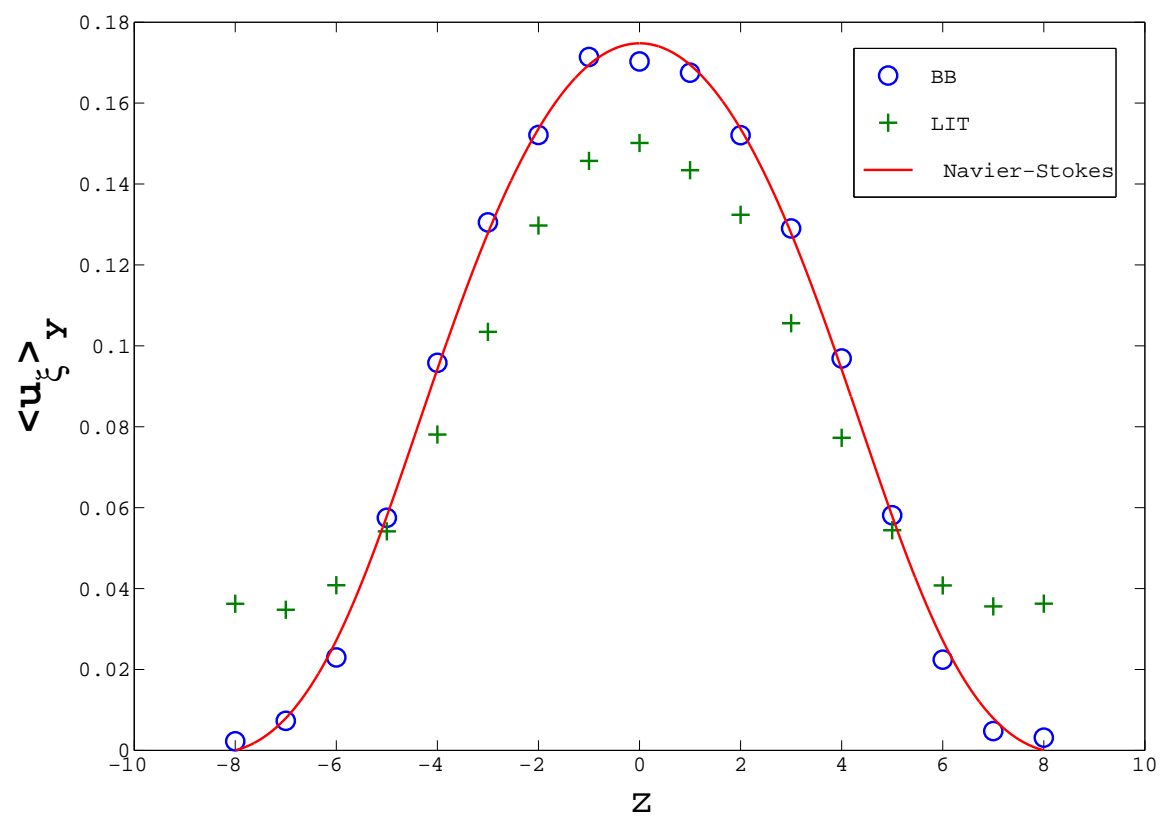

Figure 3.5: Cross-section velocity profiles downstream of the 40 percent stenosis at B with $\operatorname{Re}=4$ 


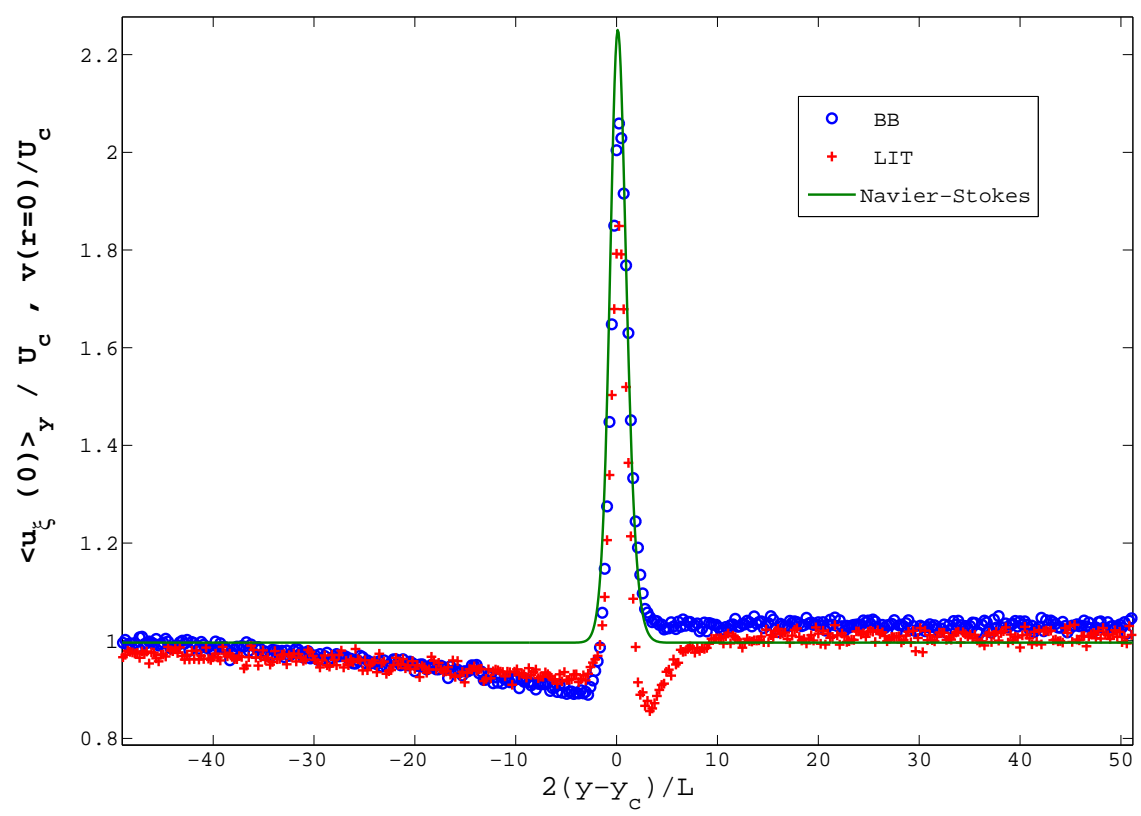

Figure 3.6: Centerline velocity profiles for 40 percent stenosis with $\mathrm{Re}=7$

Reynolds number. Figure 3.14 shows the cross-section velocity profiles downstream of the constriction. The slip is clearly seen in the case of LIT.

\subsection{Additional tests}

For $M P C$ simulations, plots of $x, y$ and $z$ components of momentum of the particle system are shown as a function of time, and temperature of the system as a function of time in Figures 3.15 to 3.18. It can be seen that the $x$ and $z$ components of momentum of particle system fluctuate about zero, and the $y$ component of momentum becomes constant after the initial transients have died out. The temperature of the system also remains constant with small fluctuations after dissipation of initial transients showing that the thermostat is working properly. 


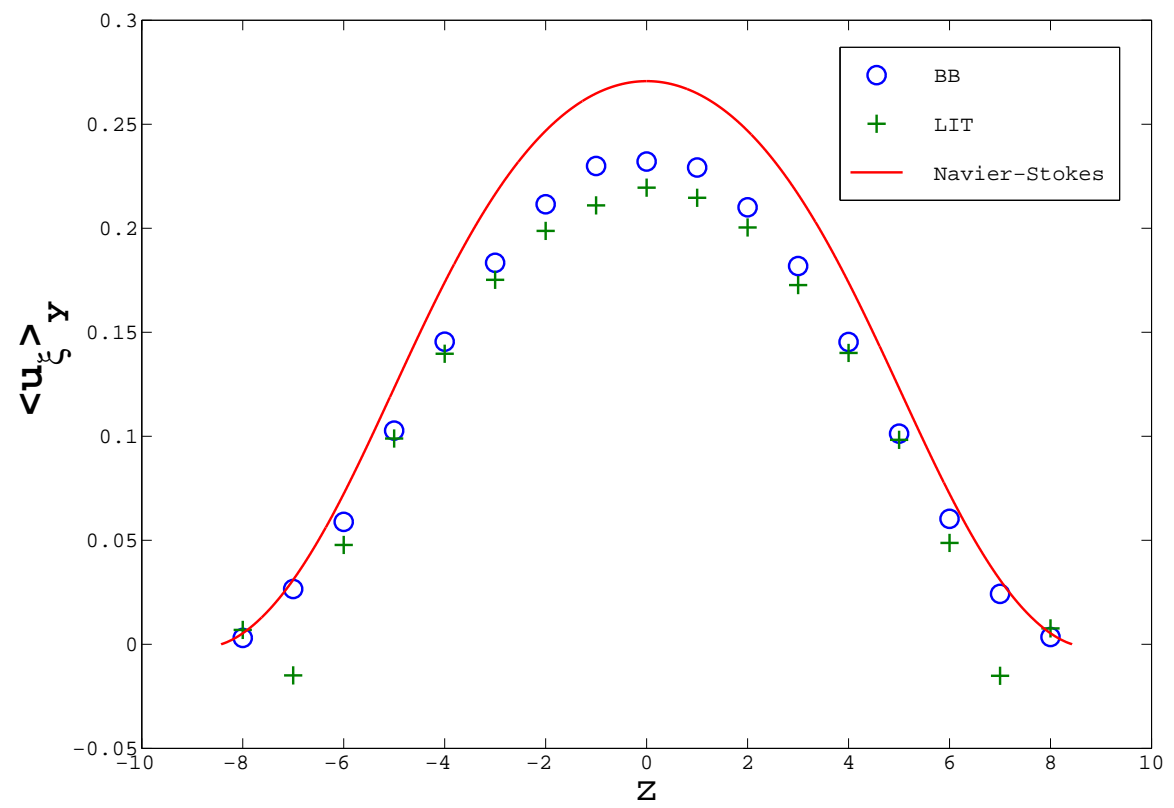

Figure 3.7: Cross-section velocity profiles upstream of the 40 percent stenosis at A with $\mathrm{Re}=7$ 


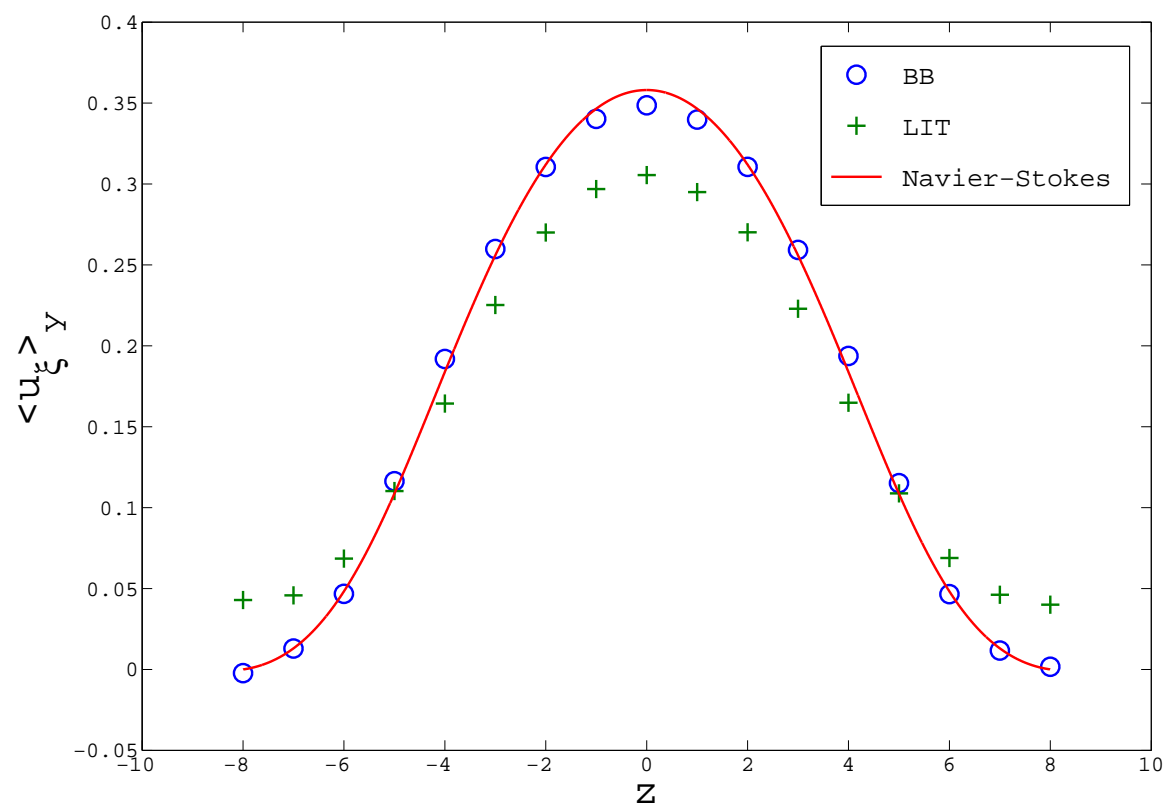

Figure 3.8: Cross-section velocity profiles downstream of the 40 percent stenosis at B : $\mathrm{Re}=7$ 

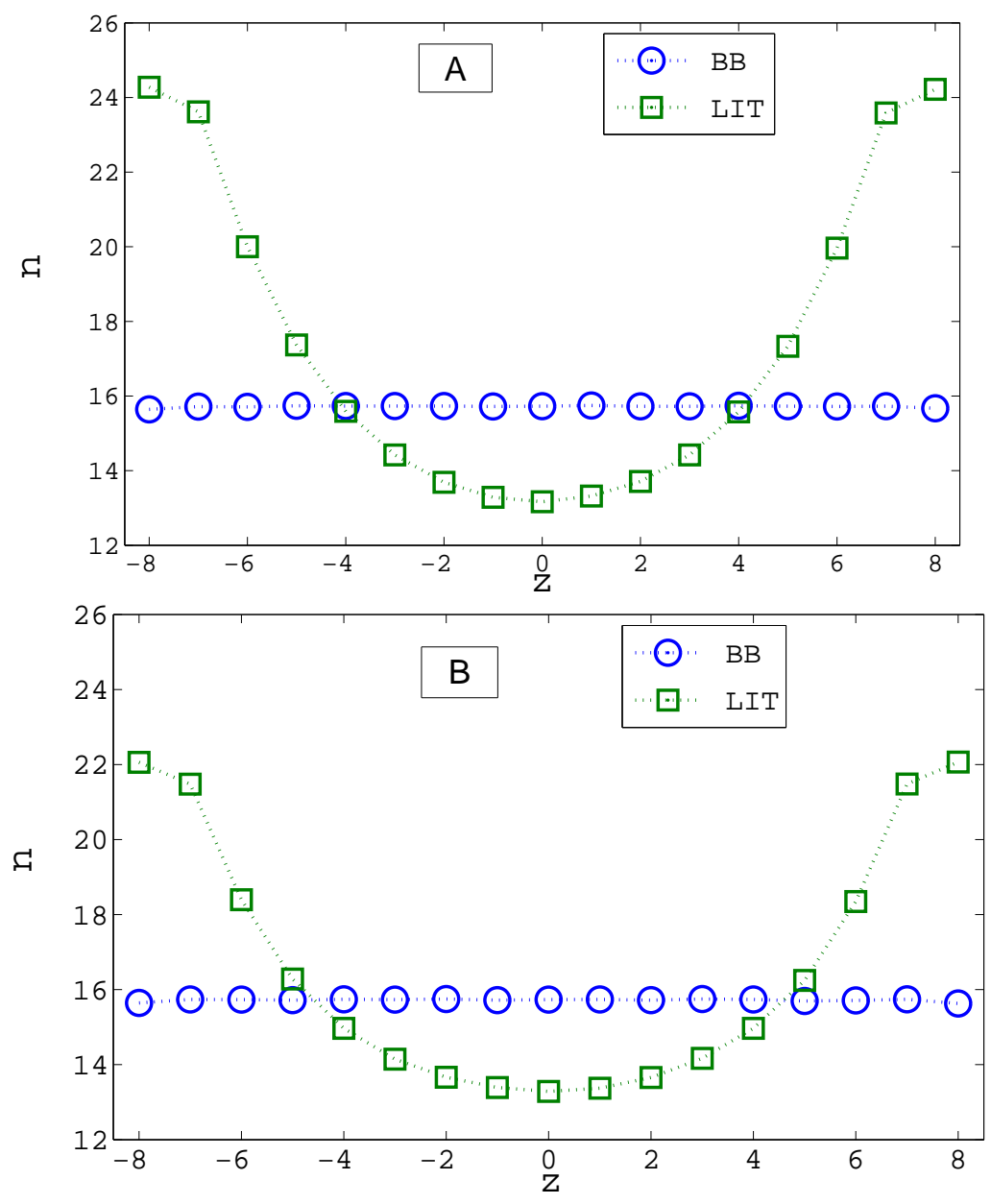

Figure 3.9: Cross-section density profiles (A) upstream of $y_{c}=226.5$ at point $\mathrm{A}$ and (B) downstream at point $\mathrm{B}$ for the 40 percent stenosis :Re $=7$ 


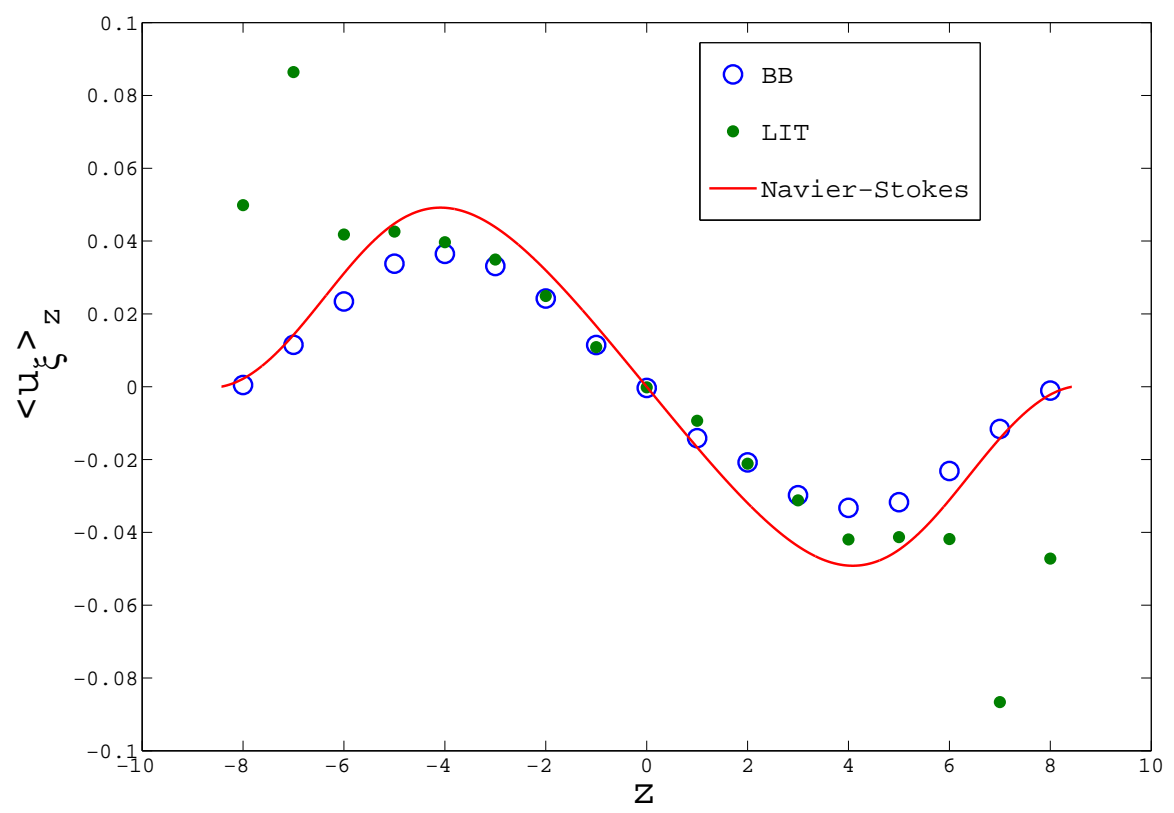

Figure 3.10: Radial velocity plot upstream of the 40 percent constriction at $\mathrm{A}: \mathrm{Re}=7$

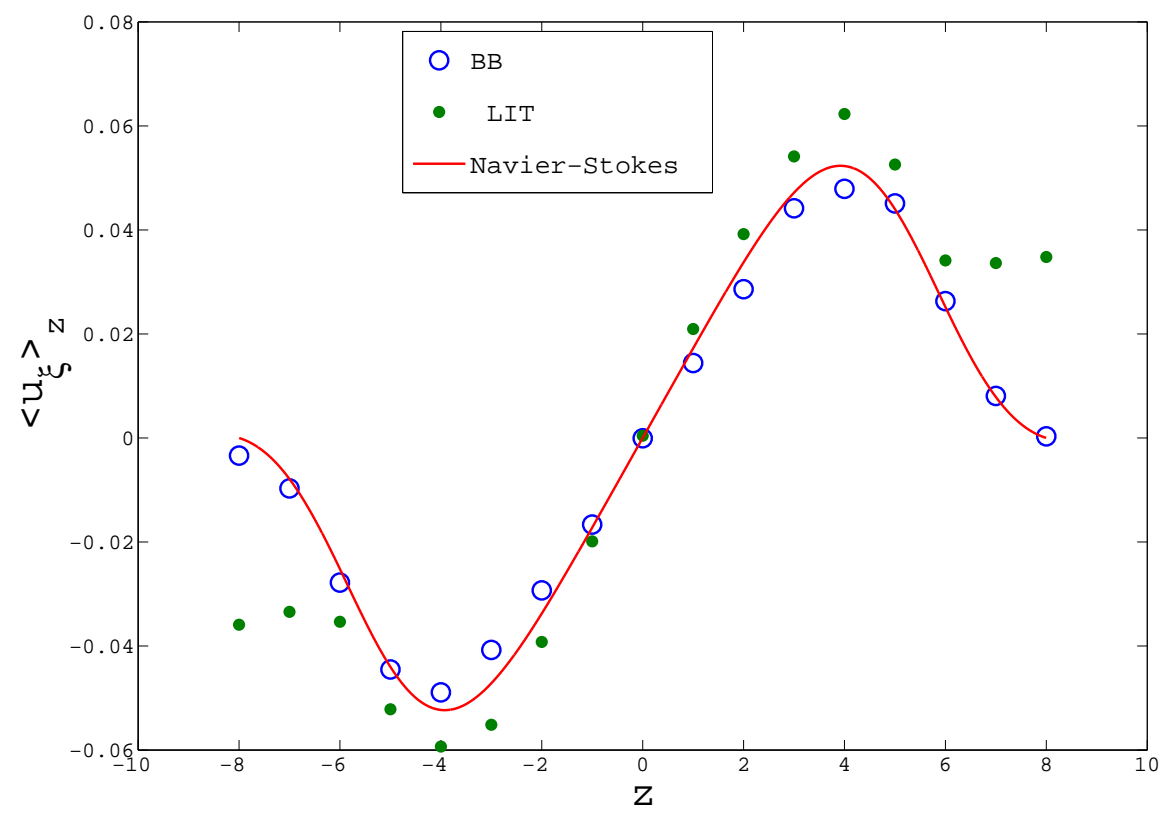

Figure 3.11: Radial velocity plots downstream of the 40 percent constriction at $\mathrm{B}: \mathrm{Re}=7$ 


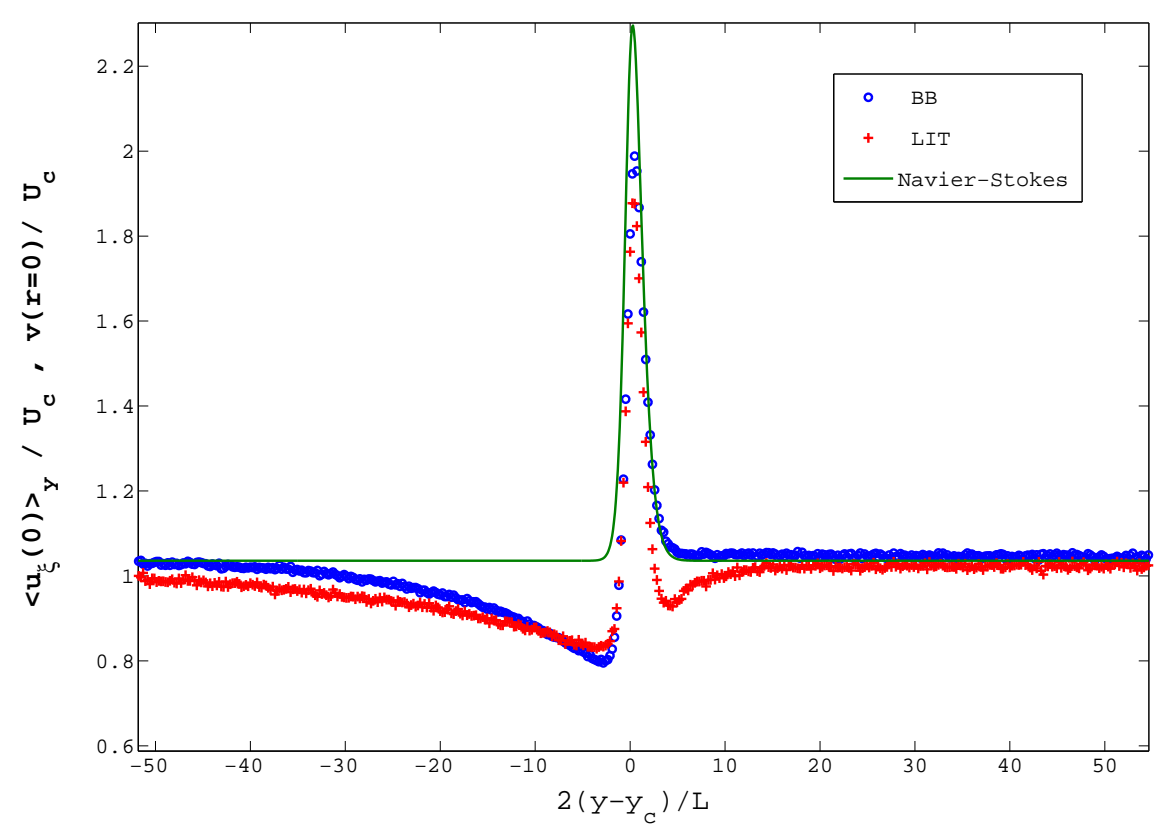

Figure 3.12: Centerline velocity profiles for 40 percent stenosis : $R e=15$

\subsection{Contour plots}

Contour plots are shown for $R e=7$ to visualize the overall flow pattern, Figure 3.19. A nice laminar axisymmetric jet can be seen in the stenotic region. The contour plots for $R e=4$ and $R e=15$ look similar and hence are not presented here.

\subsection{LIT Comparison}

To investigate the extent of the dip in the centerline velocity profiles (observed in the postconstriction region) in the case of $L I T$, plots of scaled centerline velocities as a function of scaled axial coordinate are presented in Figure 3.20. The figure shows that for a given degree of constriction $\left(s_{c}=0.2\right)$, the dip is more severe for the lower Reynolds number. Figure 3.21 shows that for a given Reynolds number $(R e=7)$, the drop in the centerline velocity in the pre-stenotic region or (upstream) is more severe for higher degrees of constriction. In order to investigate the observed drop in centerline velocities upstream of the constriction, scaled centerline density profiles are presented in Figures 3.22 and 


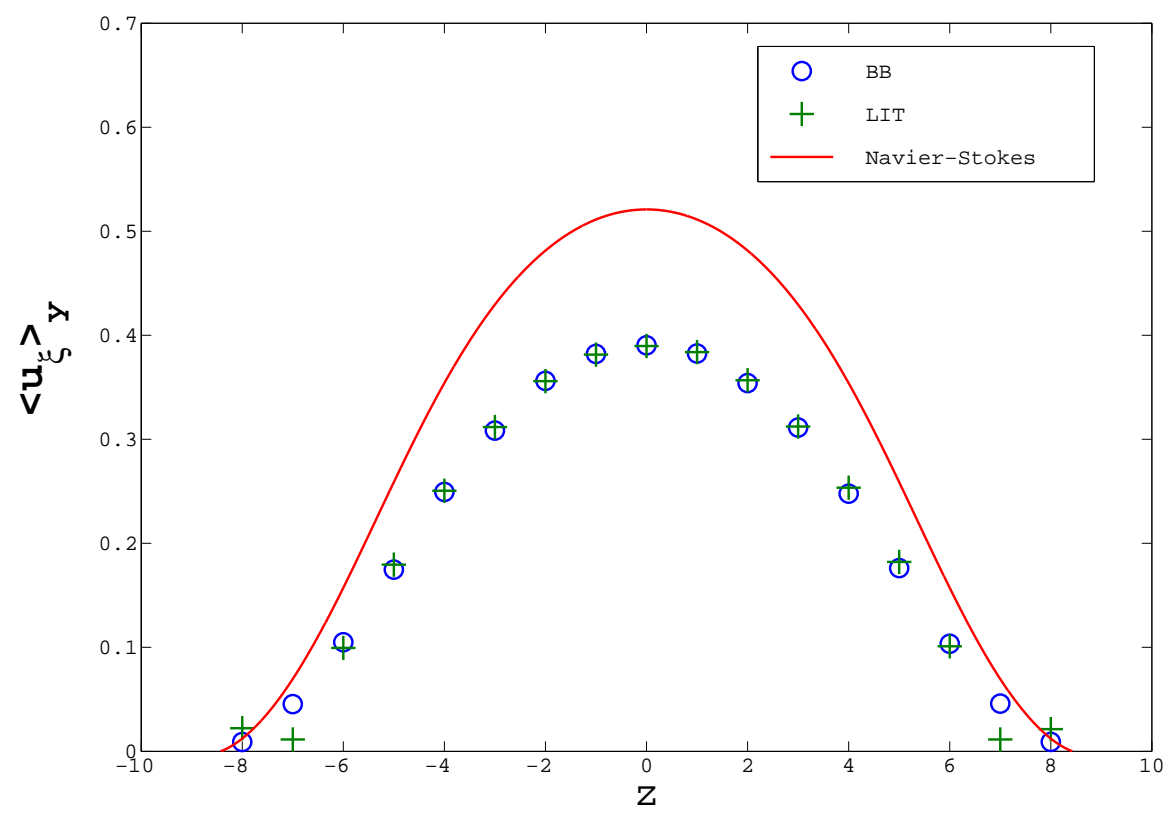

Figure 3.13: Cross-section velocity profiles upstream of the 40 percent stenosis at A : $\mathrm{Re}=15$ 


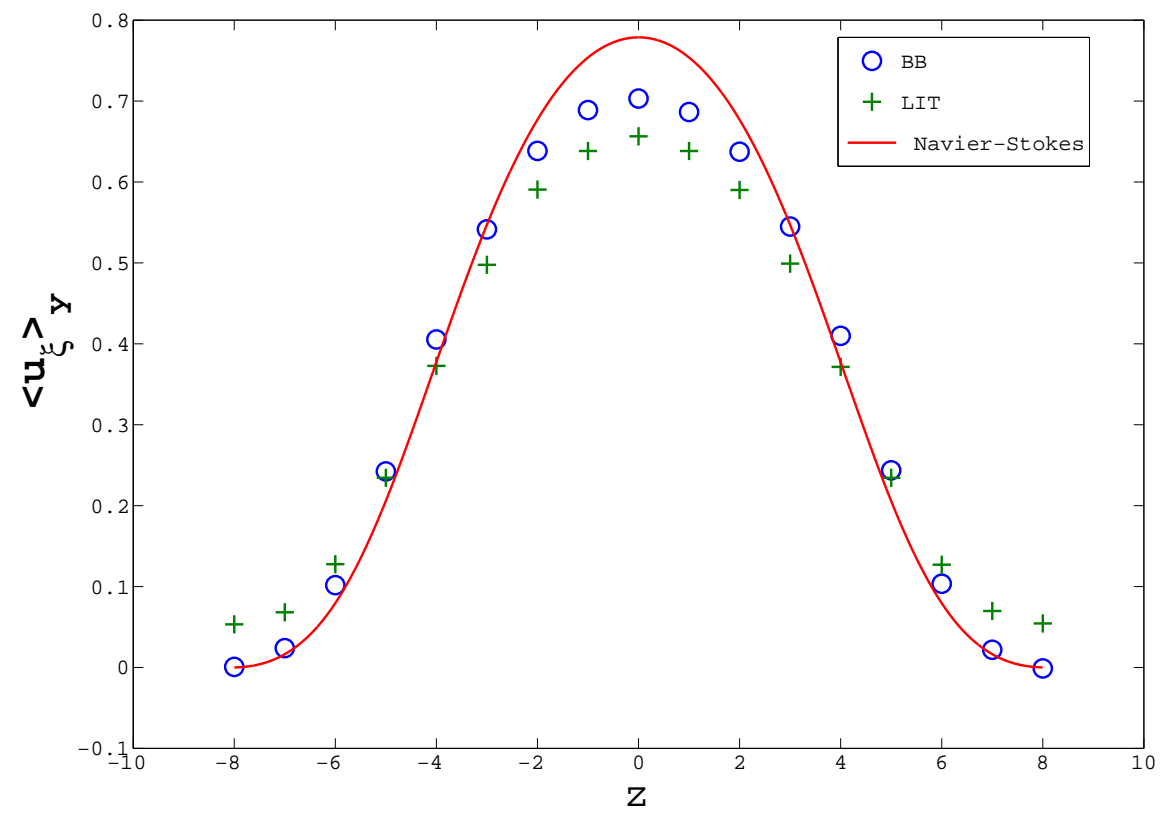

Figure 3.14: Cross-section velocity profiles downstream of the 40 percent stenosis at B : $\operatorname{Re}=15$ 


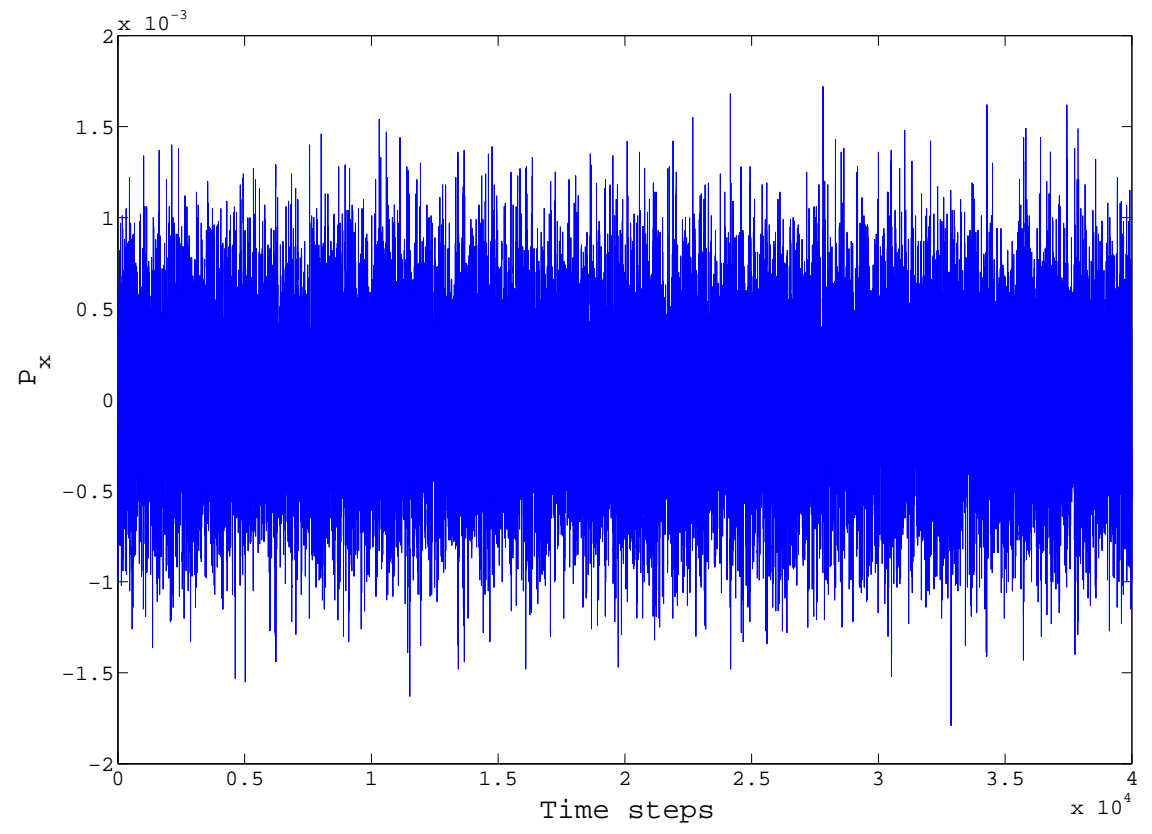

Figure 3.15: $x$-component of momentum as a function of time

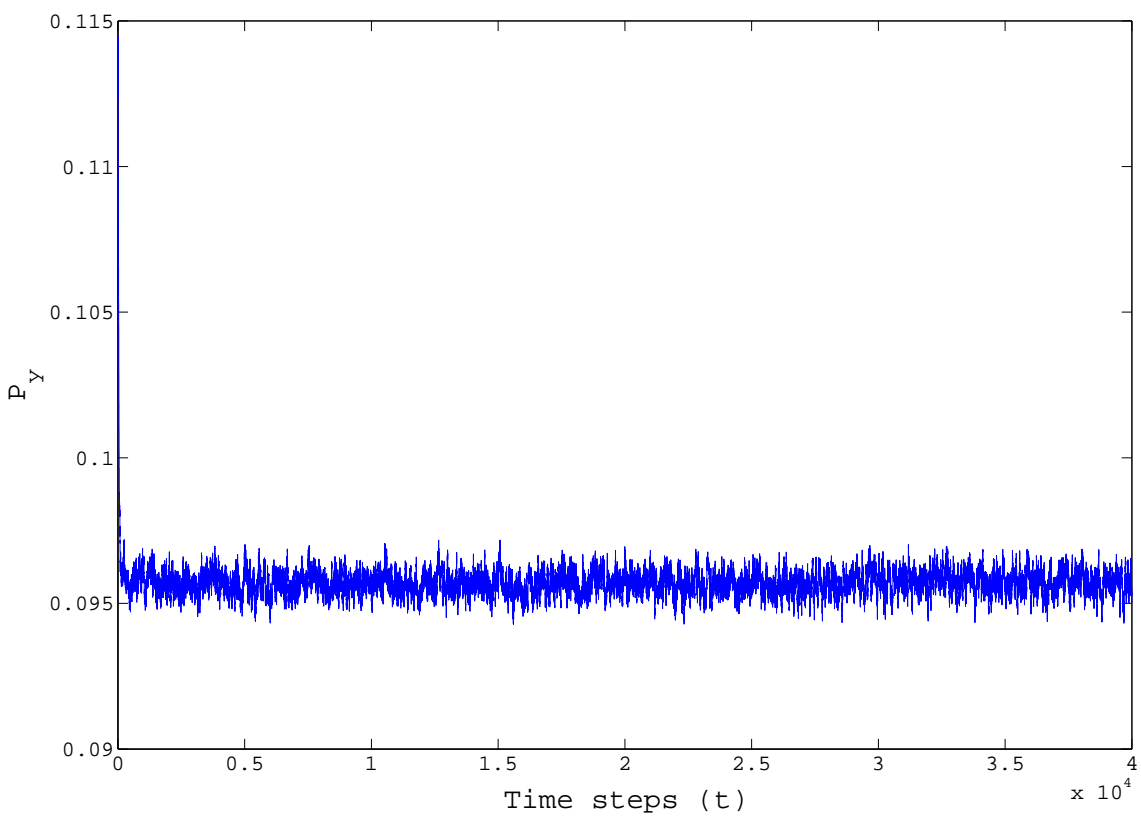

Figure 3.16: $y$-component of momentum as a function of time 


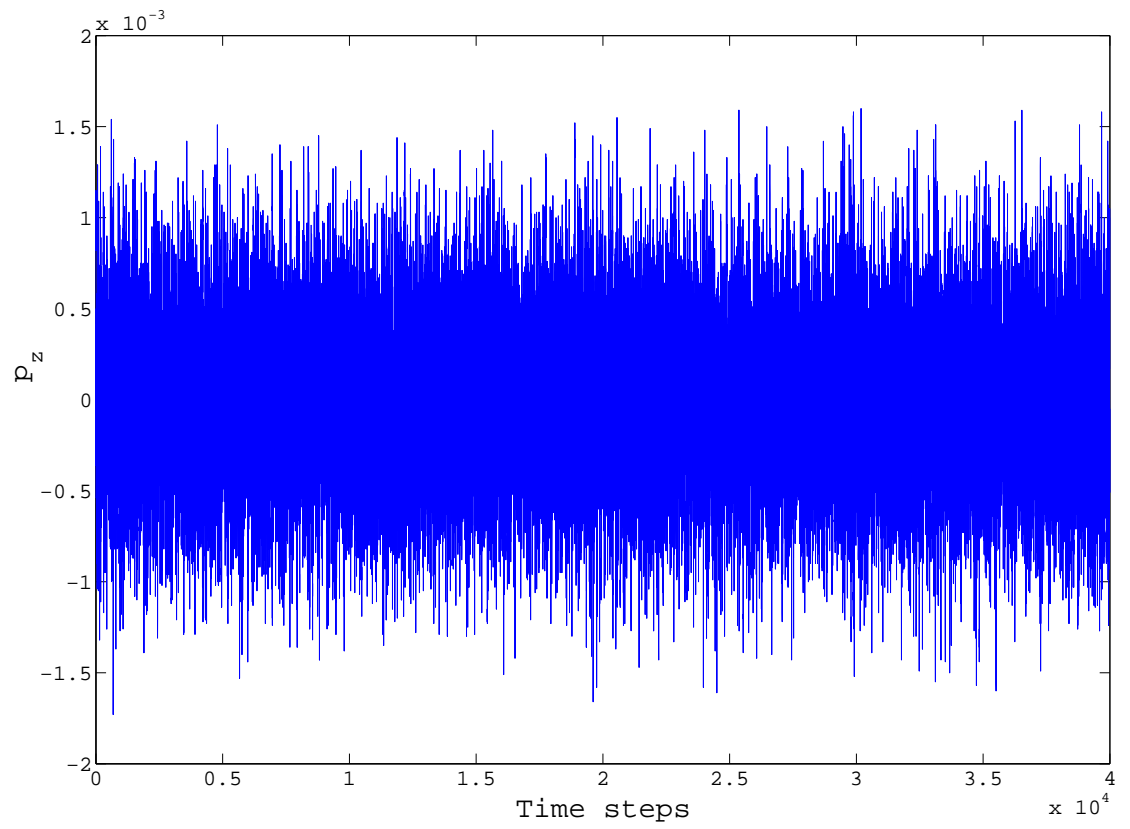

Figure 3.17: $z$-component of momentum as a function of time

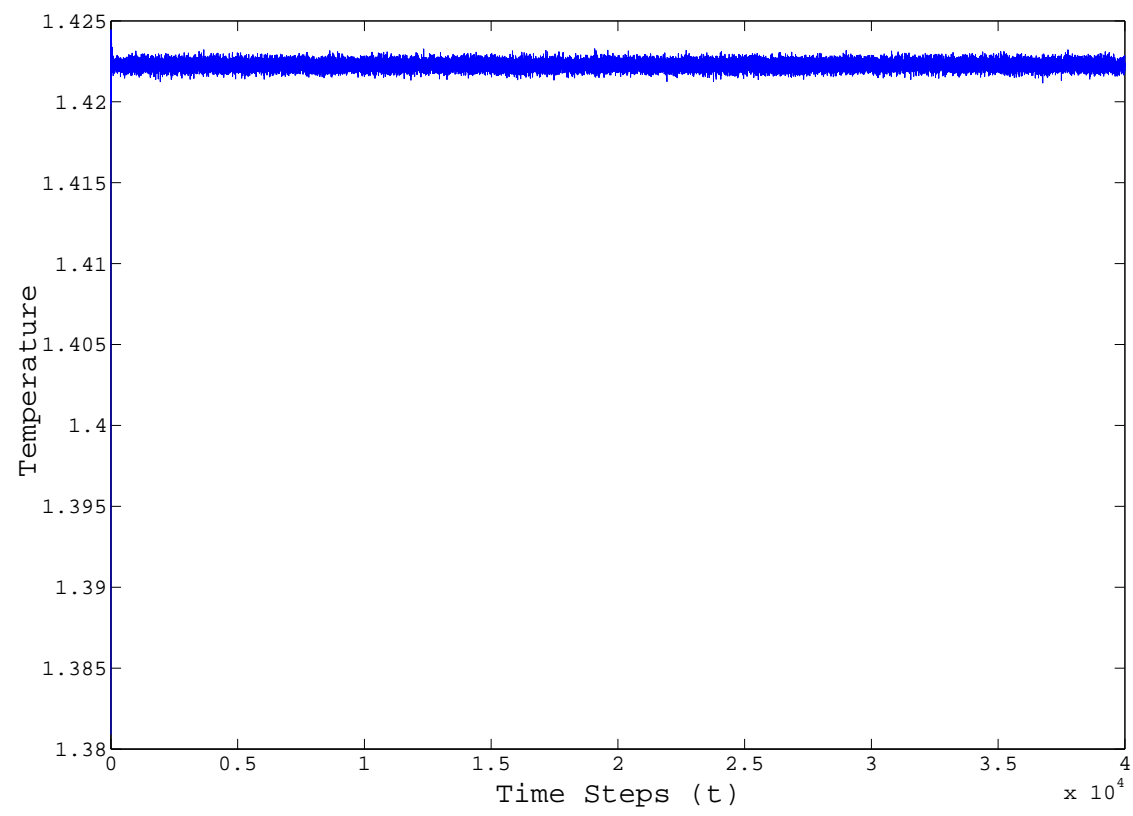

Figure 3.18: Temperature as a function of time 


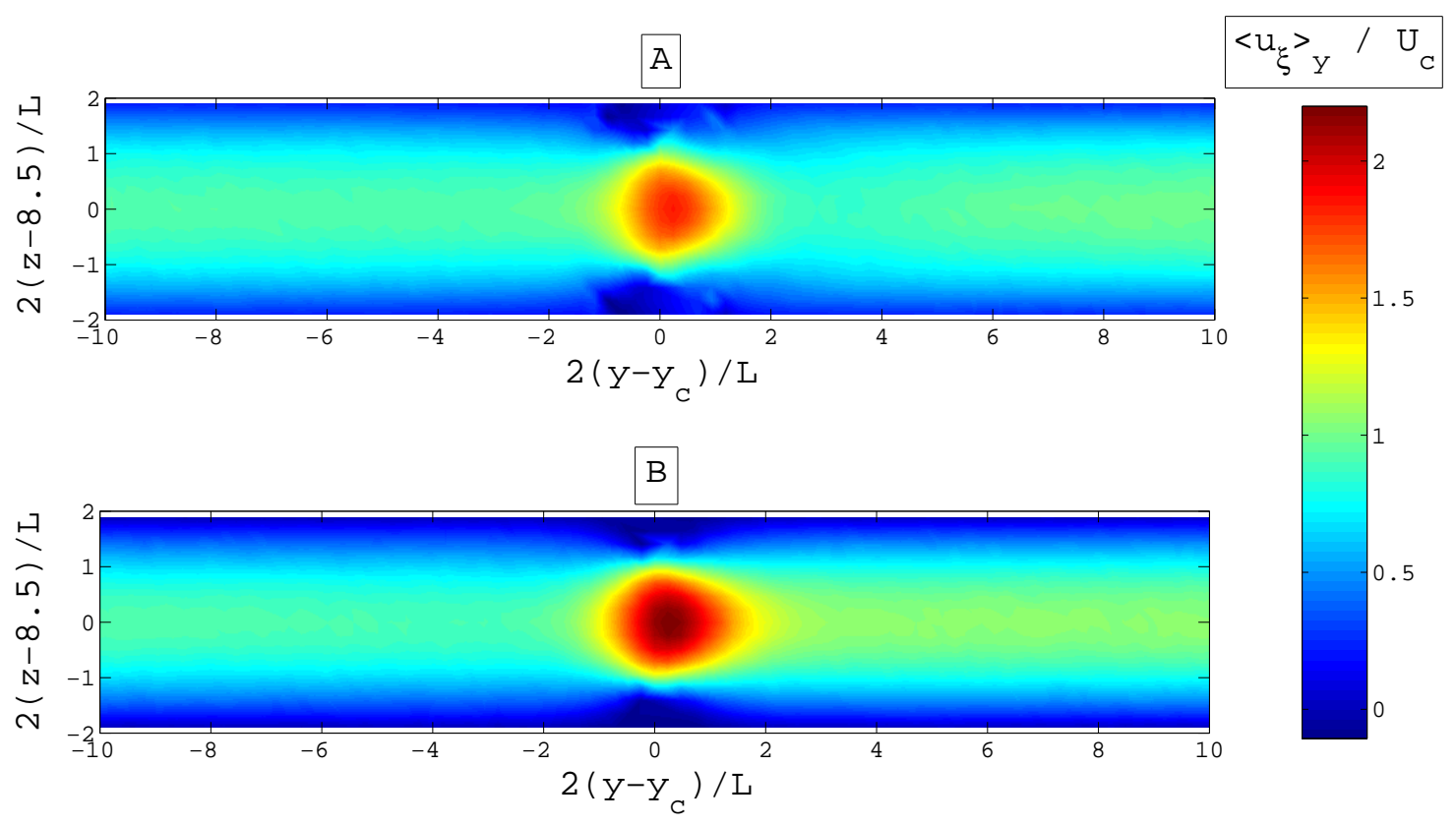

Figure 3.19: Velocity contour plots, (A) using LIT and (B) using BB boundary conditions with a 40 percent constriction : $\mathrm{Re}=7$ 


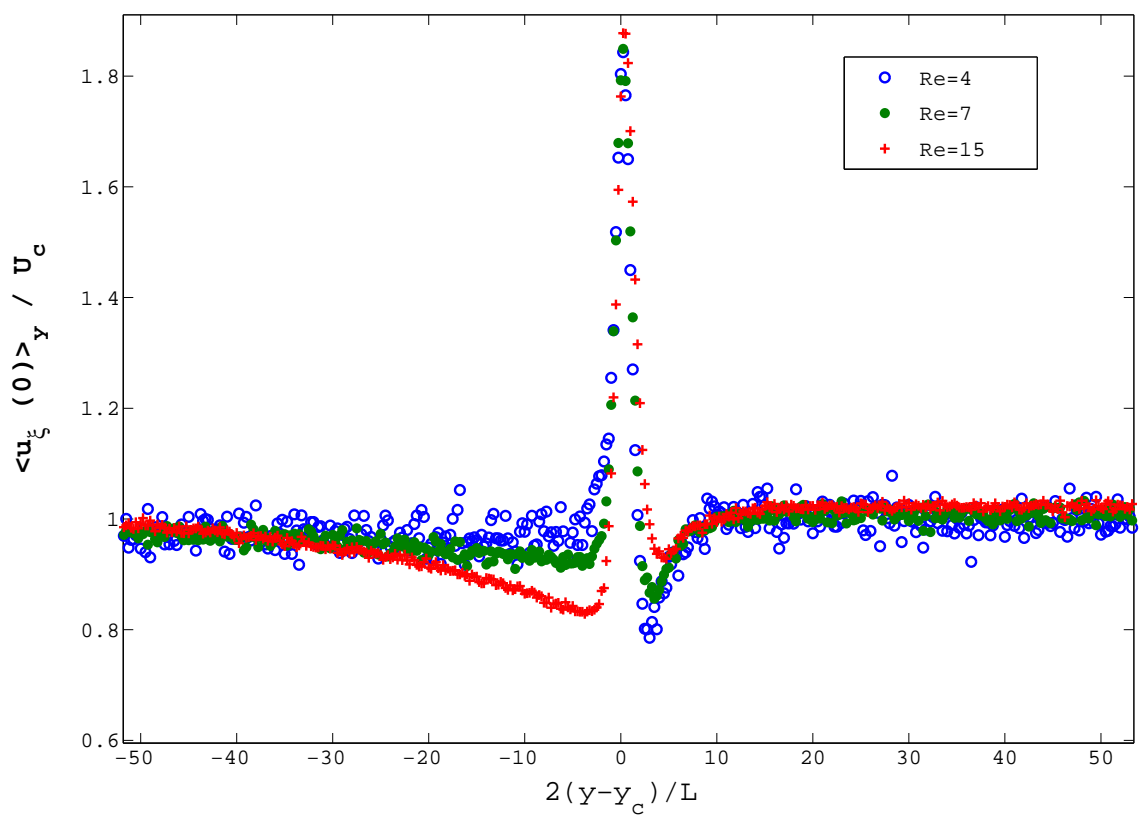

Figure 3.20: Centerline velocity profiles for LIT, 40 percent stenosis

3.23 for different values of Reynolds numbers. It can be seen that, in both the cases of $L I T$ and $B B$, the density upstream increases with increasing Reynolds number. This could be a compressibility effect and might lead to the observed decrease of centerline velocity upstream of the constriction.

\subsection{Summary of Key Findings}

- $M P C$ dynamics using $B B$ agrees well with incompressible Navier-Stokes (no-slip) for a cylinder without constriction.

- Both the boundary conditions used in MPC simulations ( $B B$ and $L I T)$ show deviations from the incompressible Navier-Stokes in the pre-stenotic and post-stenotic region.

- In the case of LIT, for a given constriction, the post-stenotic differences (dip) in centerline velocity profiles increase with decreasing Reynolds number. 


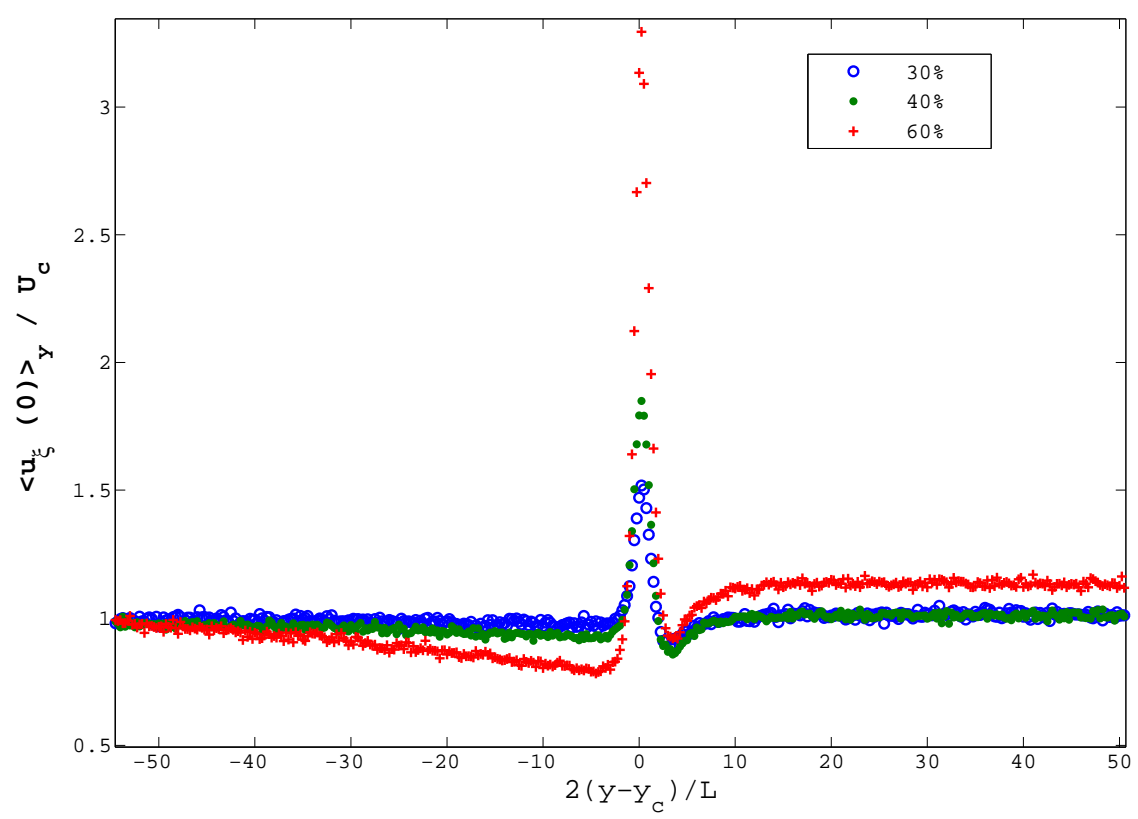

Figure 3.21: Centerline velocity profiles in case of LIT : $\operatorname{Re}=7$

- In case of LIT, for a given Reynolds number, the drop in the centerline velocity in the pre-stenotic region, increases with increasing the degree of constriction

- In a given constriction, the drop in centerline velocity in the pre-stenotic region increases with increasing Reynolds number for both boundary conditions.

- The cross-section velocity profiles obtained using $L I T$ and $B B$ show different flow profiles at the entrance of the constriction: negative velocities are observed near the wall in the case of $L I T$.

- In case of the LIT, particle density is higher near the wall and in case of $B B$, the density profile is fairly uniform across the cross-section.

- The density along the centerline increases with increasing Reynolds number possibly being the reason for the drop in the centerline velocity upstream of the constriction.

- The radial flow profiles in case of $B B$ and $L I T$ show differences near the wall regions 


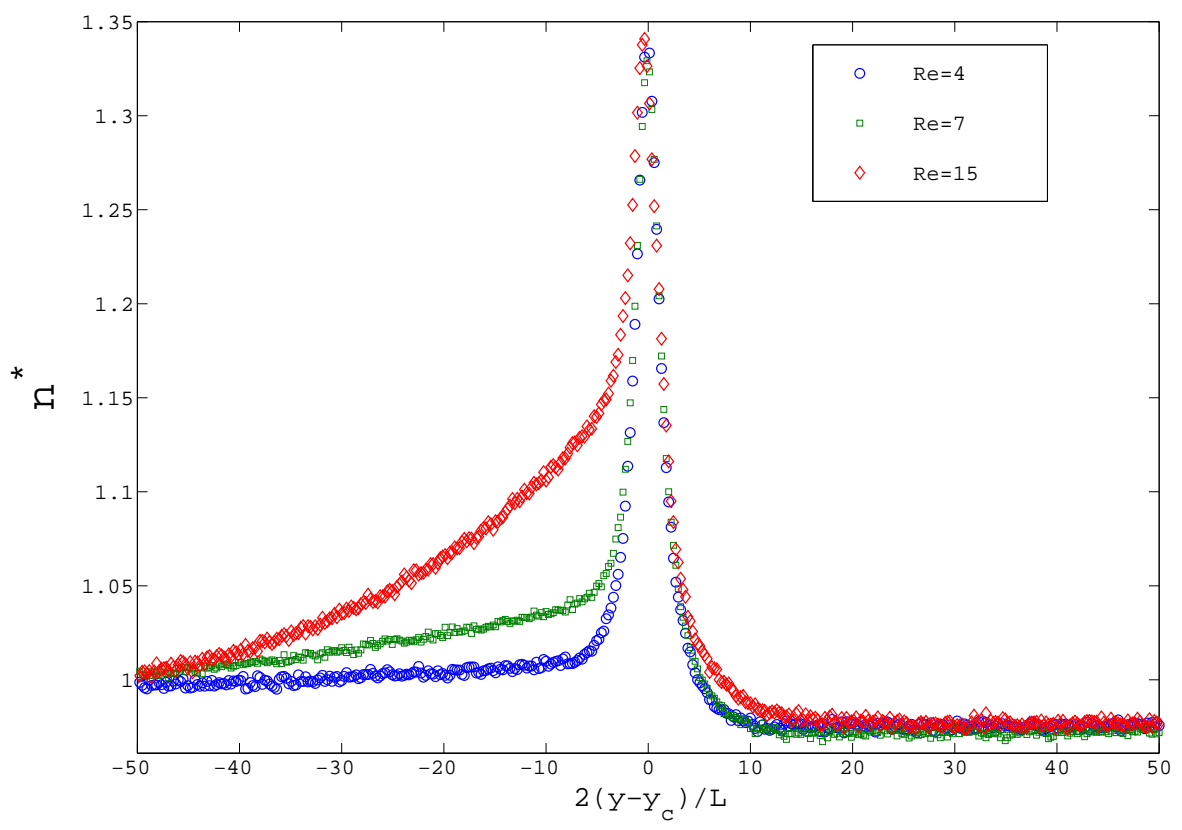

Figure 3.22: Scaled density along centerline as a function of scaled axial coordinate for $L I T$ 


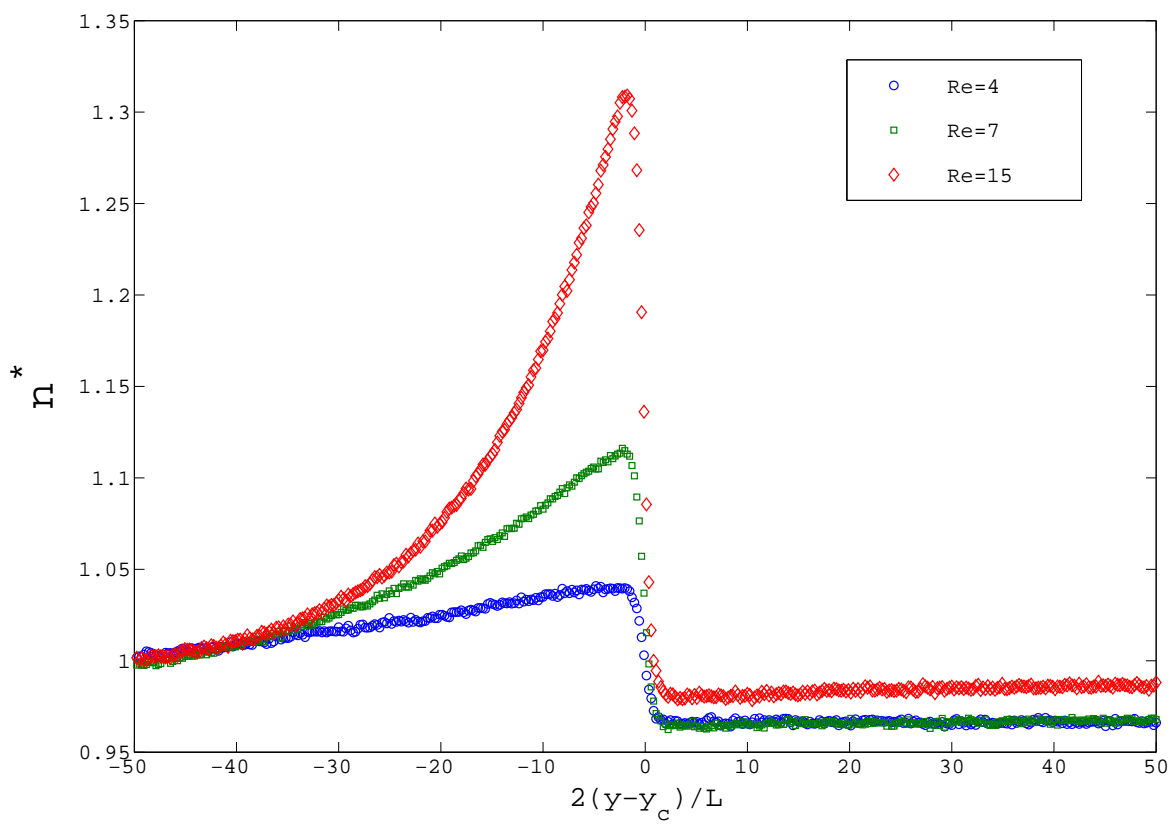

Figure 3.23: Scaled density along centerline as a function of scaled axial coordinate for the $B B$ rule 
- The radial velocity profiles obtained using $B B$ are in good agreement to those of Navier-Stokes equations.

\subsection{Discussion}

A brief discussion of the key findings now follow. The discussion mainly revolves around the observed pre-and post-stenotic differences in centerline velocity profiles, the observed negative velocities upstream of the constriction and the density profiles in both the cases and the radial velocity profiles.

The observed post-stenotic dip in the case of LIT may be a consequence of slip or a combination of slip and viscous dissipation. Since the LIT boundary condition is a dissipative boundary condition, it may modify the properties of the fluid. More rigorous investigations are needed in the future to resolve this issue. The presence of negative velocities in case of the LIT for lower Reynolds numbers considered in this work, (at location A in Figure 2.2) has not been reported elsewhere for the same geometry. The absence of negative velocities at higher Reynolds number for LIT (location A in Figure 2.2) could be a consequence of slip and compressibility. Increasing the Reynolds number means increasing the flow rate and hence the slip.

In case of LIT, since the tangential component of particle velocity is lost at the wall, the particles tend to reside near the wall, which leads to the formation of a layered structure, and the momentum in this layer is constantly being rearranged by the incoming flow momentum. This could possibly be the reason for differences in the cross-section density profiles reported in Figure 3.9. The radial velocity profiles obtained using both these boundary conditions have significantly different shapes. In case of LIT radial velocities near the wall are non-zero. The radial velocity profiles obtained using $B B$ are in good agreement with Navier-Stokes equations. The slip in case of LIT may cause these differences. In both these cases, the radial velocity profiles have symmetric shapes except a non-zero velocity near the walls in case of $L I T$. The radial flow profile in case of $B B$ is qualitatively in good agreement to that of axisymmetric lattice Boltzmann method reported in [23] by Zhou.

The Mach number defined in equation (2.34) seems to play an important role in observed decrease of the centerline velocities upstream of the constriction as seen in 


\begin{tabular}{|c|c|c|}
\hline Reynolds numbers & Centerline velocity in constriction & Local Mach number \\
\hline \hline 4 & 0.17 & 0.098 \\
\hline 7 & 0.36 & 0.27 \\
\hline 15 & 0.72 & 0.42 \\
\hline
\end{tabular}

Table 3.1: Local Mach numbers in the constriction

Figures 3.6 and 3.12. The non-dimensional speed of sound in a $M P C$ fluid is given by the simple expression $\sqrt{3 k_{B} T / m}$ and in this simulation is simply equal to $\sqrt{3}=$ 1.73. For the ranges of Reynolds numbers considered in these simulations for the case of $40 \%$ constriction, the local Mach numbers in the constricted region are computed by using equation (2.34) and are given in the table 3.1. It can be seen that, as the Reynolds number increases, the local Mach number also increases. Please note that for an incompressible fluid, the Mach number is less than one $(M a<<1)$, and when $M a \approx 1$ the compressibility effects become dominant. It is very well known that when compressible fluids encounter the sudden contraction, the mass flow rate decreases [25]. The rapid increase of density upstream of the constriction along the centerline is shown in Figures 3.22 and 3.23. The compressibility effects in case of flow past a cylinder using $M P C$ dynamics have been observed for Mach number as low as 0.17 [19]. The equation of state for the $M P C$ fluid is an ideal gas equation and hence compressibility effects are built in. The increased density upstream could cause the decrease in mass flow rate, and hence in the centerline velocity. The slip may also affect the centerline velocity, but it is not yet clear exactly how. 


\section{Chapter 4}

\section{Conclusion and Future Work}

\subsection{Summary}

In Chapter 1, the biological motivations of this study have been briefly discussed. Since $M P C$ dynamics can be extended to model chemical reactions where particle numbers can fluctuate, this feature of $M P C$ dynamics makes it more suitable to simulate red blood cell aggregation and break-up in presence of shear flow and possibly clarify the yield stress issue. The slip at the wall has been reported in the viscometric measurements and may also affect the measurements of yield stress [14]. However, simulations can help to validate the viscometric data and possibly help to understand the effects of slip on yield stress if any. The possibility of slip in flows through vascular constrictions is also discussed. Since the red blood cell aggregation has been reported in presence of the constrictions, it is necessary to simulate red blood cell aggregation and break-up explicitly. It is argued that in order to simulate red blood cell aggregation in constricted channels using $M P C$ dynamics, it is first necessary to test $M P C$ dynamics in the same geometry without aggregation using no-slip and slip boundary conditions.

Different particle-based simulation methods such as lattice Boltzmann, dissipative particle dynamics, direct simulation Monte-Carlo and multiparticle collision dynamics were also briefly discussed in the Introduction. The collision step in $M P C$ dynamics is numerically more efficient than those of other methods mentioned above, and is also very easy to implement numerically. To test the $M P C$ dynamics algorithm, the case of a cylinder without constriction was considered. Simulations of flow through the cylinder 
were performed using $B B$ and $L I T$ boundary conditions. The known case of Poiseuille flow was recovered using $M P C$ dynamics. The gravity driven flow was adapted with periodic boundary conditions along the flow direction. The velocity profiles obtained using this method are very stable and in good agreement with the theoretical Poiseuille flow profile.

Next an axisymmetric cosine shaped geometry was taken as an idealized model of a vascular constriction. Simulations of steady flow through this geometry were performed using $B B$ and LIT boundary conditions for three different Reynolds numbers. The LIT boundary conditions showed interesting differences in the centerline and cross-section velocity profiles. Finally, the results of $M P C$ simulations are compared to those of the incompressible Navier-Stokes equations solved using the finite element package COMSOL Multiphysics. The results are found to be in good qualitative agreement.

\subsection{Conclusion}

The $M P C$ dynamics has been successfully applied to simulate steady flows through an axisymmetric constricted cylinder. The $B B$ boundary conditions agree well with the solutions of incompressible Navier-Stokes (no-slip) for a cylinder without a constriction. Both boundary conditions show deviations from the incompressible Navier-Stokes, in the pre-and post-stenotic regions. The density variations due to the constriction could lead to a drop in the centerline velocity as observed in the pre-stenotic regime. The observed dip in centerline velocity (in the post-constriction region) in case of LIT could be a consequence of slip. The cross-section density profiles in case of $B B$ and LIT differ significantly. In case of $L I T$, the particle density is higher near the wall and in case of $B B$, the density profile is fairly uniform across the cross-section. The radial velocity profiles in case $B B$ and $L I T$ show differences near the wall. The difference between radial velocity profiles in $B B$ and $L I T$ could also be the consequence of slip. The radial velocity profiles obtained using $B B$ are in good agreement with Navier-Stokes (no-slip). The presence of negative velocities upstream of the constriction (point A) in case of LIT at low values of Reynolds number is not yet fully understood. The major conclusions of this study are,

- Cumulative averaging gives macroscopic no-slip in case of $B B$ without using artificial tricks. 
- The $B B$ boundary condition should be used to model blood flow if no-slip boundary condition is to be used.

- The LIT boundary condition can be used to incorporate slip.

\subsection{Future Work}

The most immediate future work is to simulate flows through the same geometry using Navier-Stokes equations with slip at the wall and compare the results with LIT. The comparison of the reported results with compressible Navier-Stokes would also be required. Recently a thermodynamically consistent particle-based model for fluid dynamics with continuous velocities and non-ideal gas equation of state has been constructed [34]. In this model the $M P C$ collision rule is modified to mimic hard sphere collisions on a coarse grained level. The model is developed to minimize the compressibility effects in $M P C$ dynamics. Such a model could be well suited for dense liquids. It would be interesting to simulate flows through constrictions using this model and to compare it with the work presented in the previous chapter. The effect of length of the stenosis on the velocity profiles should be studied using the boundary conditions employed in this work. The degree of slip can be varied using $\lambda$ in equation (2.23). It would be very interesting to see the effect of different values of $\lambda$ on the centerline velocities in case of LIT. The postulate that the dip in the post-constriction region is a consequence of slip could be explicitly verified by varying $\lambda$.

The MPC algorithm used in this work does not conserve angular momentum. The angular momentum conserving MPC dynamics has been constructed recently [35] and found to be useful to model suspensions containing finite size particles with rotational component of motion. An interesting application of angular momentum conserving $M P C$ dynamics is to simulate flows of suspensions in a Couette rheometer. The Couette rheometer is basically a rotating co-axial rheometer. The sample under consideration is placed between two concentric cylinders. The inner cylinder is attached to the torque transducer of the unit centered in the outer cylinder. The outer cylinder is rotated by using a motor and the shear stress is measured from 
the resulting torque. In this set-up a slip has been reported [14] to occur at the cylinder walls and may affect the yield stress measurements. In the angular momentum conserving MPC dynamics, the collision rule needs to be modified to include angular momentum. Computationally it is easy to implement and requires knowledge of the moment of inertia tensor for a given geometrical size of the particle. In these simulations, the shear rate can be varied to obtain the shear stress. The relation between shear stress and shear rate is called the constitutive relation and is linear for Newtonian fluids and, in general non-linear for non-Newtonian fluids. The constitutive relation can be obtained from such a simulation. The comparison between a numerically obtained constitutive equation to that of experimental data may help to quantify a yield stress as well as effects of slip on the yield stress. The aggregation of particles with constant aggregation rate in presence of flow would be the next step. In this case, more species of particles with different masses need to be included in the simulation along with the reactive collisions [2]. The shear rate dependent aggregation of particles is a challenge since the functional form of shear dependent aggregation rates is not known. Numerically, the relative velocity differences can be used to simulate shear rate dependent aggregation and break-up. The flows through other physiological complex geometries such as bifurcation of vessels and inflated blood vessels would be some other interesting applications to study using $M P C$ dynamics.

The voyage of science is never-ending, for the sake of space and time I take a pause here by quoting Albert Einstein, who once said that "The most incomprehensible thing about our universe is, it is comprehensible." I appreciate the simplicity in the complexity of nature and salute an imagination of mother nature. 


\section{Appendix A}

All simulations with the MPC dynamics use non-dimensional parameters. In order to make a connection with relevant length and time scales in physical systems, it is necessary to convert the non-dimensional parameters into dimensional ones. In these simulations, 17 cells are taken along the $z$ direction, each of size one in nondimensional units. The width of 17 cells along $z$ corresponds to a diameter of 4 $\mathrm{mm}$, which gives a dimensional cell size $a^{*}=0.235294117 \mathrm{~mm}$. All the dimensional quantities are denoted with asterisks. The non-dimensional energy of the system is

$$
k_{B} T=\frac{k_{B}^{*} T^{*}}{m^{*}\left(a^{*}\right)^{2} /\left(\tau^{*}\right)^{2}} .
$$

The temperature is taken to be $T^{*}=300 K$, mass $m^{*}=3 \times 10^{-17} \mathrm{~kg}$ and the Boltzmann constant $k_{B}^{*}=1.3806503 \times 10^{-23} \mathrm{~J} / \mathrm{K}$. The substitution of these parameters in equation (1) gives the dimensional time step to be $\tau^{*}=0.019699219$ sec. The dimensional centerline velocity is then obtained as,

$$
U_{c}^{*}=U_{c} \frac{a^{*}}{\tau^{*}}
$$

The dimensional centerline velocity in the uncostricted region for $g=0.005(R e \approx$ 7) works out to $2.18811 \mathrm{~mm} / \mathrm{s}$. 


\section{References}

[1] Anatoly Malevanets and Raymond Kapral. Mesoscopic multiparticle collision model for fluid flows and molecular dynamics. Lect. Notes. Physics, SpringerVerlarg Berlin Heidelberg, 640:116-149, 2004.

[2] Katrin Rohlf. Theoretical studies on blood flow in small vessels. PhD thesis, University of Waterloo, 2002.

[3] Katrin Rohlf. Stochastic phase space description for reactions that change particle numbers. Journal of Mathematical Chemistry, 45:17-25, 2009.

[4] Kweon, Dong-Guk-Paeng, Min Joo Choi, and Kirk Shung. Ultrasonic observation of blood disturbance in a stenosed tube, effects of flow acceleration and turbulence downstream. Ultrasound in Medicine and Biology, 34:114-122, 2008.

[5] M.J.Kang, H-S Ji, and S.J. Lee. In-vitro study of haemodiluted blood flow in sinusoidal microstenosis. Proceedings of The Institution of Mechanical Engineers, Part H, Journal of Engineering in Medicine, 224:17-25, 2009.

[6] Sonu Varghese, Steven Frankel, and Paul Fischer. Direct numerical simulation of stenotic flows.part 1.steady flow. J.Fluid Mech, 582:253-280, 2007.

[7] J.S. Stroud, S.A. Berger, and D. Saloner. Influence of stenosis morphology through severely stenotic vessels: Implications for plaque rupture. Journal of Biomechanics, 33:433-455, 2000.

[8] J.S.Stroud and S.A.Berger. Numerical analysis of blood flow through severly stenotic carotid artery bifurcation. Journal of Biomechanical Engineering, 124:9-20, 2002. 
[9] S.A Ahmed and D.P Giddens. Velocity measurements in steady flow through axisymmetric stenoses at moderate reynolds numbers. Journal of Biomechanics, 16:505-516, 1983.

[10] Yves Nubar. Blood flow,slip and viscometry. Biophysical Journal, 11:252-264, 1971.

[11] Daniel Hershey and Sung Joon Cho. Blood flow in rigid tubes, thickness and slip velocity of plasma film at the wall. Journal of Applied Physiology, 27:27$32,1965$.

[12] Atushi Nakano, Yasuhiko Sugi, and et al. Velocity profiles of pulsatile blood flow in arterioles with bifurcation and confluence in rat mesnetary measured by particle image velocimetry. JSME International Journal, Series C, 2005.

[13] J.C. Misra and G.C. Shit. Role of slip velocity in blood flow through stenosed arteries, a non-newtonian model. Journal of Mechanics in Medicine and Biology, World scientific publishing, 7:337-353, 2007.

[14] Catherine Picart, Jean-Michel Piau, and H.Gilliard. Human blood shear yield stress and its hematocrit dependence. J.Rheology, The Society of Rheology, 42:1-12, 1997.

[15] Biswas Devajyoti. Blood flow models: A Comparative Study. Mittal Publishers, 2004.

[16] P. J. Hoogerbrugge and J. M. Koelman. Simulating microscopic hydrodynamic phenomena with dissipative particle dynamics. Europhysics Letters, 19:155$160,1992$.

[17] G.A. Bird. Molecular Gas Dynamics and Direct Simulation of Gas Flows. Clarendon Press, Oxford, 1994.

[18] Anatoly Malevanets and Raymond Kapral. Mesoscopic model for solvent dynamics. Journal of Chemical Physics, 110:8605, 1999.

[19] A. Lamura and G. Gompper. Numerical study of flow around a cylinder using multiparticle collision dynamics. Eur.Phys.J E, 9:477-485, 2002.

[20] E. Allahyarov and G. Gompper. Mesoscopic solvent simulations: multiparticle collision dynamics of three dimensional flows. Physical Review E, 66:036702, 2002. 
[21] Daniel A.P. Reid, H. Hildenbrandt, J.T. Padding, and C.K. Hemelrijk. Flow around fishlike shapes studied using multiparticle collision dynamics. Physical Review E, 79:046313, 2009.

[22] Matthew T Downton and Holger Stark. Simulation of model microswimmer. Journal of Physics, Condensed Matter, 21:204101, 2009.

[23] Jian Guo Zhou. Axisymmetric lattice Boltzmann method. Physical Review E, 78:036701, 2008.

[24] J.R.Darias, M.Quiroga, E.Medina, P.J.Colmenares, and R.Paredes. Simulation of suspensions in constricted geometries by dissipative particle dynamics. Molecular Simulation, 29:443-449, 2003.

[25] Landau and Lifshitz. Course of Theoretical Physics, Vol.6,. Elsevier Publication, 2008.

[26] Denis J. Evans and Gary P. Morris. Shear thickening and turbulence in simple fluids. Physical Review Letters, 56:2172-2175, 1986.

[27] J.T Padding and A.A Louis. Hydrodynamic interactions and brownian forces in colloidal suspensions:coarse graining over time and length scales. Physical Review E, 74:031402, 2006.

[28] Jonathan K.Whitmer and Erik Luijten. Fluid-solid boundary conditions for multiparticle collision dynamics. Journal of Physics, Condensed Matter, IOP Publishing, 2009.

[29] T. Ihle and D.M. Kroll. Stochastic rotation dynamics,formalism-I, Galileaninvariance, and Green-Kubo relations. Physical Review E, 67:066705, 2003.

[30] T. Ihle and D.M. Kroll. Stochastic rotation dynamics: A Galilean invariant mesoscopic model for fluid flow. Physical Review E, 63:020201, 2001.

[31] Martin W. Tysanner and Alejandro L. Garcia. Non-equilibrium behaviour of equilibrium reservoirs in molecular simulations. International Journal For Numerical Methods In Fluids, 48:1337-1349, 2005.

[32] H. Noguchi, N. Kikuchi, and G. Gompper. Particle based mesoscale hydrodynamic techniques. European Physics Letters, 78:016706, 2007.

[33] Version 3.2. Comsol Multiphysics User's Guide. COMSOL, 2005. 
[34] T. Ihle, E. Tüzel, and D.M. Kroll. Consistent particle-based algorithm with a non-ideal equation of state. Europhysics Letters, 73:664-670, 2006.

[35] Hiroshi Noguchi and Gerhard Gompper. Transport coefficients of offlattice mesoscale hydrodynamics simulation techniques. Physical Review E, 78:016706, 2008. 